\title{
EULER PRODUCTS ASSOCIATED TO METAPLECTIC AUTOMORPHIC FORMS ON THE 3-FOLD COVER OF GSp(4)
}

\author{
THOMAS GOETZE
}

\begin{abstract}
If $\phi$ is a generic cubic metaplectic form on $\operatorname{GSp}(4)$, that is also an eigenfunction for all the Hecke operators, then corresponding to $\phi$ is an Euler product of degree 4 that has a functional equation and meromorphic continuation to the whole complex plane. This correspondence is obtained by convolving $\phi$ with the cubic $\theta$-function on GL(3) in a Shimura type RankinSelberg integral.
\end{abstract}

\section{INTRODUCTION}

Suppose $\phi$ is a metaplectic automorphic form of minimal level on the 3 -fold cover of $\mathrm{GSp}(4)$ that is an eigenfunction of all the Hecke operators. If $\phi$ has any non-zero Whittaker coefficients, then $\phi$ is called generic. In this case, this paper will show that there is a Dirichlet series in the Whittaker coefficients of $\phi$ that has a formulation as a degree 4 Euler product. Moreover, this Euler product has a meromorphic continuation to the whole complex plane. This association of the Euler product with $\phi$ will be obtained via a Shimura type Rankin-Selberg integral involving $\phi$ and a $\theta$-function on the 3 -fold cover of GL(3).

Historically, the problem of associating an Euler product which has meromorphic continuation and functional equation with a metaplectic automorphic form originated with the work of Shimura [Shi]. More specifically, suppose $f(z)=\sum a(n) q^{n}$ is a holomorphic modular form of half-integral weight $k / 2$, which is an eigenform of the Hecke operators $T_{p^{2}}$, i.e. $T_{p^{2}} f=\lambda_{p} f$. Then via a Rankin-Selberg integral of the form

$$
\int f(z) \overline{\theta(z)} E(z, s) d z
$$

where $\theta(z)$ is a classical theta function and $E(z, s)$ is an integral weight Eisenstein series, Shimura obtains an Euler product of the form

$$
\prod_{p}\left(1-\lambda_{p} p^{-s}+p^{k-2-2 s}\right)^{-1}
$$

The analytic continuation and functional equation of this Euler product follow from the similar properties of $E(z, s)$ in $(0.1)$.

Bump and Hoffstein [BH2] have subsequently extended these techniques of [Shi] to GL(3) by finding a Rankin-Selberg integral of a metaplectic automorphic form on the 3 -fold cover of GL(3) which produces an Euler product of degree 3. Just as in [Shi], this Euler product is shown to have meromorphic continuation to the whole

Received by the editors November 14, 1995 and, in revised form, May 21, 1996.

1991 Mathematics Subject Classification. Primary 11F55, 11 F30. 
complex plane and to have a functional equation under $s \mapsto 1-s$. The integral which represents this Euler product involves the $\theta$-function on the 3 -fold cover of GL(3) over the field $\mathbb{Q}\left(e^{2 \pi i / 3}\right)$, which has been studied independently by Proskurin [Pr] and Bump and Hoffstein [BH1]. In addition, Bump and Hoffstein [BH2] have conjectured that Euler products with meromorphic continuation and functional equation may be obtained by convolving metaplectic automorphic forms on the $n$-fold cover of GL $(r)$ against $\theta$-functions on the $n$-fold cover of GL $(n)$. This was carried out in [BH3] in the case $r=2$ and $n>2$.

Friedberg and Wong [FrW] have also used Shimura's method to associate an Euler product to a generic metaplectic automorphic form on the double cover of the symplectic group GSp(4). They have found an integral (inspired by Novodvorsky's GSp $(4) \times \mathrm{GL}(2)$ convolution) involving a metaplectic automorphic form on the double cover of $\operatorname{GSp}(4)$, the $\theta$-function on the double cover of $\operatorname{GL}(2)$, and a (non-metaplectic) Eisenstein series on GL(2), that yields a degree 5 Euler product. This Euler product is shown to have meromorphic continuation and a functional equation, and furthermore it has the same local Euler factors as the $L$-function of an automorphic form on GSp(4). As in [Shi, BH2], the Euler product found by Friedberg and Wong is explicitly constructed from the Whittaker coefficients of the metaplectic automorphic form.

Alternatively, Flicker [Fli], Kazhdan and Patterson [KaP2], and Flicker and Kazhdan [FliKa] have used the trace formula to generalize [Shi] by showing that (in many situations) there exists a correspondence between metaplectic automorphic forms and (non-metaplectic) automorphic forms. Indeed, Shimura actually proves in [Shi] that the Euler product (0.2) is the $L$-function of a holomorphic integral weight modular form. In using the trace formula, however, explicit information about the interplay between the metaplectic Fourier coefficients and the corresponding $L$-functions (see (0.2)) is not obtained.

If a generalized Shimura correspondence does exist between generic metaplectic and non-metaplectic automorphic forms, then the associated Euler products obtained by Bump-Hoffstein, Friedberg-Wong, and this paper will be the $L$-functions of the corresponding non-metaplectic forms. There is evidence that the degree 4 Euler product obtained in this paper is the $L$-function of an automorphic form on GSp(4). Savin [Sa] has shown that there is an algebra isomorphism between the local Iwahori Hecke algebra of GSp(4) and the local Iwahori Hecke algebra on the 3 -fold cover of GSp(4). This suggests that if a Shimura correspondent exists in this situation, it should be an automorphic form on GSp(4). Since there is a representation of degree 4 on the $L$-group of $\operatorname{GSp}(4)$, automorphic forms on $\operatorname{GSp}(4)$ will have natural $L$-functions with Euler products of degree 4 . In this sense, having a degree 4 Euler product is consistent with Savin's results.

The main results of this paper will be found in Theorems 3.1 and 5.1, which are summarized as follows:

Main Theorem. Suppose $\phi$ is a generic metaplectic cusp form of minimal level on the 3-fold cover of $\mathrm{GSp}(4)$ that is an eigenfunction of all the Hecke operators. Then there is a degree 4 Euler product, with a meromorphic continutation, which can be explicitly constructed from the Whittaker coefficients of $\phi$. This association is realized as a Shimura type Rankin-Selberg integral of $\phi$ against an Eisenstein series induced from a $\theta$-function on the 3-fold cover of $\mathrm{GL}(3)$. 
The form of the Shimura integral used in the proof is a translation from the adelic version of the non-metaplectic GSp $(4) \times$ GL(3) convolution that Bump has considered [B2]. Note, as Bump has, that the non-metaplectic convolution was already available via "Method B" of Gelbart and Piatetski-Shapiro [GeP-S], although it was not explicitly constructed there. Although a translation from the non-metaplectic to the metaplectic works in the case considered here, it is not always possible to obtain a metaplectic convolution in this manner.

The Shimura type integral considered in the proof of the Main Theorem may be naturally regarded as a 3 -fold $\mathrm{GSp}(4) \times \mathrm{GL}(3)$ convolution. Thus, [FrW] and the results of this paper suggest that generalizations of Shimura's techniques to $n$-fold covers of $\mathrm{GSp}(4)$ will yield Euler products as $n$-fold $\mathrm{GSp}(4) \times \mathrm{GL}(n)$ convolutions. In fact, from the evidence obtained for $n=2$ and $n=3$ the following conjecture may be made.

Conjecture. Let $n$ be a positive integer, and suppose $\phi$ is a generic metaplectic cusp form on the $n$-fold cover of GSp(4) which is an eigenfunction of all the Hecke operators. Then there is an Euler product associated to $\phi$ that has a meromorphic continuation and a functional equation under $s \mapsto 1-s$. This association can be explicitly realized as a Shimura type Rankin-Selberg convolution of $\phi$ with an $n$-fold $\theta$-function on GL( $n)$. If $n$ is even this Euler product will be of degree 5 , while if $n$ is odd a degree 4 Euler product will be obtained.

Generalizations of Shimura's techniques to $n$-fold $G \times \mathrm{GL}(n)$ convolutions should also be possible for higher rank groups $G$. The conjecture in [BH2] mentioned above, along with the results in $[\mathrm{FrW}]$ and this paper, suggest that it is possible to obtain an Euler product from the convolution of a generic metaplectic cusp form on an $n$-fold cover of $\operatorname{GL}(r)$ or $\operatorname{GSp}(4)$ with a $\theta$-function on the $n$-fold cover of $\operatorname{GL}(n)$. Although there is little evidence, it is natural to expect that this may hold for more general groups than just GL $(r)$ and GSp(4). In this sense, the expectation is that an Euler product with meromorphic continuation and functional equation may be constructed from a metaplectic automorphic form $\phi$ on any reductive algebraic group $G$. This construction would likely be a $G \times \mathrm{GL}(n)$ convolution obtained via an integral involving $\phi$ and an $n$-fold $\theta$-function on GL( $n)$. The non-metaplectic analogues of these conjectured convolutions have been studied in [Gi], [GeP-S], and [So].

The rest of the paper is organized as follows. Section 1 contains notation and preliminaries needed throughout the paper. In Section 2, the metaplectic automorphic forms that will be used in the Shimura integral are defined. The Shimura integral is presented and evaluated to be a double Dirichlet series in Section 3. Then Section 4 provides the definition of the Hecke operators on the 3-fold cover of GSp(4) and gives an explicit description of the action of the operators on the Whittaker-Fourier coefficients of a metaplectic form. Next, using this description of the Hecke operators, the double Dirichlet series obtained in Section 3 is shown to have an Euler product in Section 5, provided the GSp(4) form is a Hecke eigenform. Finally, Section 5 ends with a brief discussion about the functional equation for the Euler product.

The results of this paper were first derived for the author's Ph.D. thesis at the University of California, Santa Cruz. As such, the author wishes to thank his Ph.D. advisor Solomon Friedberg for his many helpful consultations and suggestions 
during the preparation of both thesis and paper. Research for this paper has been supported in part by NSA grant MDA904-95-H-1053.

\section{Preliminaries}

For $n \geq 1$, let $\mathcal{Z}_{n}$ be the center of $\operatorname{GL}(n, \mathbb{C})$ and define

$$
\mathcal{H}_{n}=\mathrm{GL}(n, \mathbb{C}) / \mathcal{Z}_{n} U(n) \text {. }
$$

$\operatorname{GL}(n, \mathbb{C})$ operates on $\mathcal{H}_{n}$ via left multiplication. For notational ease the coordinates

$$
\begin{gathered}
\tau_{2}=\left(\begin{array}{cc}
1 & x_{1} \\
& 1
\end{array}\right)\left(\begin{array}{ll}
y_{1} & \\
& 1
\end{array}\right), \\
\tau_{3}=\left(\begin{array}{ccc}
1 & x_{2} & x_{3} \\
& 1 & x_{1} \\
& & 1
\end{array}\right)\left(\begin{array}{cccc}
y_{1} y_{2} & & \\
& y_{1} & \\
& & 1
\end{array}\right),
\end{gathered}
$$

and

$$
\tau_{4}=\left(\begin{array}{cccc}
1 & x_{2} & x_{3} & x_{4} \\
& 1 & x_{1} & x_{5} \\
& & 1 & x_{6} \\
& & & 1
\end{array}\right)\left(\begin{array}{cccc}
y_{1} y_{2} y_{3} & & & \\
& y_{1} y_{2} & & \\
& & y_{1} & \\
& & & 1
\end{array}\right)
$$

will be used for $\tau_{n} \in \mathcal{H}_{n}, n=2,3,4$, where $x_{i} \in \mathbb{C}, y_{i} \in \mathbb{R}^{+}$. The choice of such coordinates for these symmetric spaces is a direct result of the Iwasawa decomposition.

For $\omega=e^{2 \pi i / 3}$, let $K=\mathbb{Q}(\omega)$. Then $K$ contains $\mu_{3}$, the group of cube roots of unity. Define $\lambda=\sqrt{-3}=1+2 \omega, e(a+b i)=e^{4 \pi i a}$, and $\mathbb{N}$ to be the norm map, i.e., for $a, b \in \mathbb{Q}, \mathbb{N}(a+b \omega)=a^{2}-a b+b^{2}$. The ring of integers in $K$ is $\mathcal{O}=\mathbb{Z}[\omega]$. The principal ideal generated by the element $m$ will be denoted by $(m)$, and the fractional ideal $(a)^{-k}=\left\{b \in \mathbb{Q}(\omega) \mid a^{k} b \in \mathcal{O}\right\}$. Also define

$$
\begin{aligned}
\Gamma_{n} & =\operatorname{SL}(n, \mathcal{O}), \\
\Gamma_{n}(3) & =\left\{\gamma \in \Gamma_{n} \mid \gamma \equiv I \bmod (3)\right\}, \\
\Gamma_{n, \infty} & =\left\{\left(a_{i, j}\right) \in \Gamma_{n} \mid a_{i, j}=0 \text { if } i>j, \text { and } a_{i, i}=1\right\}, \\
\Gamma_{n, \infty}(3) & =\left\{\gamma \in \Gamma_{n, \infty} \mid \gamma \equiv I \bmod (3)\right\}=\Gamma_{n}(3) \cap \Gamma_{n, \infty} .
\end{aligned}
$$

The classes of cusps $\rho_{i}$ of $\Gamma_{2}(3)$ are, by definition, double coset representatives of $\Gamma_{2}(3) \backslash \Gamma_{2} / \Gamma_{2, \infty}$ in $\Gamma_{2}$ (i.e. $\left.\Gamma_{2}=\bigcup_{i} \Gamma_{2}(3) \rho_{i} \Gamma_{2, \infty}\right)$. In particular, an available set of representatives for these double cosets is

$$
\begin{aligned}
&\left\{\left(\begin{array}{ll}
1 & 0 \\
0 & 1
\end{array}\right),\left(\begin{array}{cc}
0 & -1 \\
1 & 0
\end{array}\right),\left(\begin{array}{cc}
1 & 0 \\
\pm 1 & 1
\end{array}\right),\left(\begin{array}{cc}
1 & 0 \\
\pm \omega^{2} & 1
\end{array}\right)\right. \\
&\left.\left(\begin{array}{cc}
1 & 0 \\
\pm \omega & 1
\end{array}\right),\left(\begin{array}{cc} 
\pm(1-\omega) & -1 \\
1 & 0
\end{array}\right),\left(\begin{array}{cc}
1 & 0 \\
\pm(1-\omega) & 1
\end{array}\right)\right\}
\end{aligned}
$$

(see Section 1 of $[\mathrm{Pa}]$ ). Throughout the rest of the paper $\rho$ will denote one of these representatives.

Let $(\dot{\bullet})_{3}$ be the cubic residue symbol in $\mathcal{O}$. The following proposition will be useful throughout the rest of the paper. 
Proposition 1.1. There is a map $\kappa_{n}: \Gamma_{n}(3) \rightarrow \mu_{3}$, called the Kubota symbol, with the following properties:

(i) $\kappa_{n}$ is a homomorphism;

(ii) $\kappa_{2}\left(\begin{array}{ll}a & b \\ c & d\end{array}\right)= \begin{cases}\left(\frac{c}{d}\right)_{3} & \text { if } c \neq 0 ; \\ 1 & \text { if } c=0 ;\end{cases}$

(iii) $\kappa_{n}(\gamma)=1$, if $\gamma \in \Gamma_{n, \infty}$ and $n \geq 2$;

(iv) if $A \in \Gamma_{n-1}(3)$ and $n \geq 2$, then

$$
\kappa_{n}\left(\left(\begin{array}{cc}
A & 0 \\
0 & 1
\end{array}\right)\right)=\kappa_{n}\left(\left(\begin{array}{cc}
1 & 0 \\
0 & A
\end{array}\right)\right)=\kappa_{n-1}(A) .
$$

This has been proved in the work of Bass, Milnor and Serre [BMS] for level $(\sqrt{-3})^{3}$. To obtain the result for level 3, Proskurin suggests that the arguments in [BMS] can still be applied-see [Pr] for the details in the case that $n=3$. Alternatively, minor modifications to the work of Bump, Friedberg, and Hoffstein [BFrH2] could also be applied to improve the level.

Now the notation and definitions for $\operatorname{GL}(n, \mathbb{C})$ are complete, but similar notation and definitions are necessary for the symplectic group GSp(4). For our purposes, define

$$
G=\mathrm{GSp}(4, \mathbb{C})=\left\{g \in \mathrm{GL}(4, \mathbb{C}) \mid{ }^{t} g J g=\mu(g) J, \mu(g) \in \mathbb{C}\right\},
$$

where

$$
J=\left(\begin{array}{llll} 
& & & -1 \\
& & -1 & \\
& 1 & &
\end{array}\right)
$$

Let $\mathcal{Z}$ be the center of $G$ and define $\mathcal{H}=G / \mathcal{Z}(U(4) \cap G)$. Elements of $\mathcal{H}$ will be denoted $\tau$ with the coordinates

$$
\tau=\left(\begin{array}{cccc}
1 & x_{2} & x_{3} & x_{4} \\
& 1 & x_{1} & x_{3}^{\prime} \\
& & 1 & -x_{2} \\
& & & 1
\end{array}\right)\left(\begin{array}{cccc}
y_{1} y_{2} & & & \\
& y_{1} & & \\
& & 1 & \\
& & & y_{2}^{-1}
\end{array}\right),
$$

where $x_{3}^{\prime}=x_{3}-x_{1} x_{2}, x_{i} \in \mathbb{C}, y_{i} \in \mathbb{R}^{+}$. Next define $\Gamma(3)=\Gamma_{4}(3) \cap \operatorname{GSp}(4, \mathcal{O})$ and let $\kappa: \Gamma(3) \rightarrow \mu_{3}$ denote the restriction of $\kappa_{4}$ to $\Gamma(3)$. When an embedding of $\mathrm{GL}(2, \mathbb{C})$ in $G$ is required, the following embedding will be used: for $\gamma=$ $\left(\begin{array}{ll}a & b \\ c & d\end{array}\right) \in \mathrm{GL}(2, \mathbb{C})$, define

$$
\mathcal{J}(\gamma)=\left(\begin{array}{cccc}
1 & & & \\
& a & b & \\
& c & d & \\
& & & 1
\end{array}\right) \in G .
$$

Finally, the following lemma will be used later:

Lemma 1.2. If $9 \mid n$, for $n \in \mathcal{O}$, and $\rho \in \Gamma_{2}(3) \backslash \Gamma_{2} / \Gamma_{2, \infty}$, then

$$
\kappa\left(\mathcal{J}(\rho) \mathcal{J}\left(\begin{array}{cc}
1 & n \\
& 1
\end{array}\right) \mathcal{J}(\rho)^{-1}\right)=\kappa_{2}\left(\rho\left(\begin{array}{cc}
1 & n \\
& 1
\end{array}\right) \rho^{-1}\right)=1 .
$$


Proof. The first equality follows from Proposition 1.1 (iv). The second equality will be checked by using an explicit set of cusps, as in (1.4), and the cubic reciprocity law.

$$
\text { If } \rho=\left(\begin{array}{ll}
1 & 0 \\
0 & 1
\end{array}\right) \text {, then }
$$

$$
\kappa_{2}\left(\rho\left(\begin{array}{cc}
1 & n \\
& 1
\end{array}\right) \rho^{-1}\right)=1
$$

by Proposition 1.1 (iii). So if $c \neq 0$ and $\rho=\left(\begin{array}{ll}a & b \\ c & d\end{array}\right)$, then

$$
\kappa\left(\rho\left(\begin{array}{cc}
1 & n \\
& 1
\end{array}\right) \rho^{-1}\right)=\left(\frac{-n c^{2}}{1+n a c}\right)_{3} .
$$

So, by applying (1.6) to each of the non-identity representatives in (1.4), it suffices to compute

$$
\begin{gathered}
\left(\frac{-n}{1}\right)_{3},\left(\frac{-n}{1 \pm n}\right)_{3},\left(\frac{-n \omega}{1 \pm n \omega^{2}}\right)_{3},\left(\frac{-n \omega^{2}}{1 \pm n \omega}\right)_{3} \\
\left(\frac{-n}{1 \pm n(1-\omega)}\right)_{3},\left(\frac{n(1-\omega)^{2}}{1 \pm n(1-\omega)}\right)_{3}
\end{gathered}
$$

Now $\left(\frac{-n}{1}\right)_{3}=1$, so the lemma has been verified for the first two cusp representatives listed in (1.4).

To establish the lemma in the other cases, first recall that for any non-zero element $a^{\prime} \in \mathbb{Z}[\omega]$, there exist positive integers $\nu$ and $\mu$ such that $a^{\prime}=(1-\omega)^{\nu} \omega^{\mu} a$, for $a \in \mathbb{Z}[\omega]$ with $a \equiv \pm 1 \bmod (3)$. Then the law of cubic reciprocity for $\mathbb{Z}[\omega]$ can be stated as follows:

1. If $a, b$ are relatively prime and each is congruent to $\pm 1 \bmod (3)$, then

$$
\left(\frac{a}{b}\right)_{3}=\left(\frac{b}{a}\right)_{3}
$$

2. If $a= \pm\left(1+3\left(a_{1}+a_{2} \omega\right)\right)$, then

$$
\begin{aligned}
& \left(\frac{\omega}{a}\right)_{3}=\omega^{-a_{1}-a_{2}}, \\
& \left(\frac{1-\omega}{a}\right)_{3}=\omega^{a_{1}} .
\end{aligned}
$$

This formulation is Exercise 2.14 in Cassels and Fröhlich [CF].

Now assuming $9 \mid n$, let $x=n / 9$. By an appropriate choice of $y, z \in \mathbb{Z}[\omega]$, all the remaining cases from (1.7) are then of the form $\left(\frac{9 x y}{1+9 x z}\right)_{3}$. To compute these remaining cases write $x z=x_{1}+x_{2} \omega$ and $9 x y=(1-\omega)^{\nu} \omega^{\mu} a$, with $a \equiv \pm 1 \bmod (3)$. The choice of $y$ forces $x \equiv 0 \bmod (a)$. Then by the law of cubic reciprocity 


$$
\begin{aligned}
\left(\frac{9 x y}{1+9 x z}\right)_{3} & =\left(\frac{1-\omega}{1+3\left(3 x_{1}+3 x_{2} \omega\right)}\right)_{3}^{\nu}\left(\frac{\omega}{1+3\left(3 x_{1}+3 x_{2} \omega\right)}\right)_{3}^{\mu}\left(\frac{a}{1+9 x z}\right)_{3} \\
& =\left(\omega^{3 x_{1}}\right)^{\nu}\left(\omega^{-3 x_{1}-3 x_{2}}\right)^{\mu}\left(\frac{1+9 x z}{a}\right)_{3} \\
& =\left(\frac{1}{a}\right)_{3} \\
& =1
\end{aligned}
$$

Thus, the proof of Lemma 1.2 is complete.

\section{Cubic metaplectic automorphic forms}

Metaplectic automorphic forms are automorphic forms on metaplectic groups. As with all automorphic forms, one may study the forms either as functions on the group or as functions on the corresponding symmetric space. For this paper, the latter approach will be employed.

Definition 2.1. A cubic metaplectic automorphic form on $\mathrm{GL}(n, \mathbb{C})$ for $\Gamma_{n}(3)$ is a smooth function $\mathrm{F}$ defined on $\mathcal{H}_{n}$ and satisfying:

1. $F\left(\gamma \tau_{n}\right)=\overline{\kappa_{n}(\gamma)} F\left(\tau_{n}\right)$ for all $\gamma \in \Gamma_{n}(3), \tau_{n} \in \mathcal{H}_{n}$;

2. $F$ is an eigenfunction of all the invariant differential operators on $\mathcal{H}_{n}$;

3. there is a constant $c$ such that

$$
\left|F\left(\gamma \tau_{n}\right)\right| \leq\left(y_{1}+y_{1}^{-1}\right)^{c}\left(y_{2}+y_{2}^{-1}\right)^{c} \ldots\left(y_{n-1}+y_{n-1}^{-1}\right)^{c}
$$

for all $\tau_{n} \in \mathcal{H}_{n}, \gamma \in \Gamma_{n}$, and $\tau_{n}$ in the form given in (1.1)-(1.3).

Let $\rho$ be a cusp of $\Gamma_{2}(3), n_{1} \in(\lambda)^{-3}$, and $n_{2} \in(\lambda)^{-5}$. Then for a cubic metaplectic automorphic form $f$ on $\mathrm{GL}(3)$ for $\Gamma_{3}(3)$, define

$$
\begin{aligned}
& f_{n_{1}, n_{2}}^{\rho}\left(\tau_{3}\right) \\
& \quad=\left(\frac{2}{3^{9 / 2}}\right)^{3} \int_{\mathbb{C} /(3)} \int_{\mathbb{C} /(9)} \int_{\mathbb{C} /(3)} f\left(\left(\begin{array}{ll}
\rho & \\
& 1
\end{array}\right) \tau_{3}\right) e\left(-n_{1} x_{1}-n_{2} x_{2}\right) d x_{1} d x_{2} d x_{3} .
\end{aligned}
$$

The factor in front of this integral normalizes the Lebesgue measure on $\mathbb{C}$. It is a consequence of the local multiplicity one theorem of Shalika [Shal] that there exist Whittaker-Fourier coefficients $b_{n_{1}, n_{2}}^{\rho}$ of $f$ that satisfy the equality

$$
f_{n_{1}, n_{2}}^{\rho}\left(\tau_{3}\right)=b_{n_{1}, n_{2}}^{\rho} \mathbb{N}\left(n_{1} n_{2}\right)^{-1} W_{f}\left(\left(\begin{array}{ccc}
n_{1} n_{2} & & \\
& n_{1} & \\
& & 1
\end{array}\right)\left(\begin{array}{ccc}
y_{1} y_{2} & & \\
& y_{1} & \\
& & 1
\end{array}\right)\right),
$$

where $W_{f}$ is the non-degenerate Whittaker function associated to $f$. See Section 3 of [BH1] for an account of these Whittaker functions. 
The following lemma will be needed later.

Lemma 2.2. If $f\left(\left(\begin{array}{ccc} \pm 1 & & \\ & \pm 1 & \\ & & 1\end{array}\right) \tau_{3}\right)=f\left(\tau_{3}\right)$, then the Whittaker-Fourier coefficients $b_{n_{1}, n_{2}}^{\rho}$ of $f$ satisfy

$$
b_{n_{1}, n_{2}}^{\rho}=b_{\left|n_{1}\right|, n_{2}}^{\rho}=b_{n_{1},-n_{2}}^{\rho^{*}}
$$

where

$$
\left(\begin{array}{ll}
a & b \\
c & d
\end{array}\right)^{*}=\left(\begin{array}{cc}
a & -b \\
-c & d
\end{array}\right)
$$

Proof. This follows from the definition of the Whittaker-Fourier coefficients (2.1) and the matrix identities

$$
\left(\begin{array}{lll}
a & b & \\
c & d & \\
& & 1
\end{array}\right)\left(\begin{array}{lll}
-1 & & \\
& 1 & \\
& & 1
\end{array}\right)=\left(\begin{array}{ccc}
-1 & & \\
& 1 & \\
& & 1
\end{array}\right)\left(\begin{array}{ccc}
a & -b & \\
-c & d & \\
& & 1
\end{array}\right)
$$

and

$$
\left(\begin{array}{lll}
a & b & \\
c & d & \\
& & 1
\end{array}\right)\left(\begin{array}{lll}
1 & & \\
& -1 & \\
& & 1
\end{array}\right)=\left(\begin{array}{lll}
1 & & \\
& -1 & \\
& & 1
\end{array}\right)\left(\begin{array}{ccc}
a & -b & \\
-c & d & \\
& & 1
\end{array}\right) .
$$

For this paper, the key example of a cubic metaplectic automorphic form on GL(3) is $\Theta$, the cubic theta function. Kazhdan and Patterson have defined theta series on $n$-fold metaplectic groups as residues of Eisenstein series [KaP1]. Subsequently, Proskurin [Pr] and Bump and Hoffstein [BH1, BH2] have independently studied $\Theta$ in more detail. In particular, Bump and Hoffstein [BH1] have explicitly computed the Whittaker-Fourier coefficients $\theta^{\rho}\left(n_{1}, n_{2}\right)=\theta_{n_{1}, n_{2}}^{\rho}$ of $\Theta$ as defined in (2.1), if $\rho$ is an essential cusp (see [Pa], p. 127, for a definition of essential cusp). Although the explicit values of $\theta_{n_{1}, n_{2}}^{\rho}$ are not needed here, the following proposition will be instrumental in the following sections.

Proposition 2.3. (Bump-Hoffstein) If $n_{1}, n_{2} \in \mathcal{O}^{\times}$are $p$-adic units, then for the Gauss sum $g(a, b)$ as defined in (4.1),

(i) $\theta^{\rho}\left(n_{1} p^{k_{1}}, n_{2} p^{k_{2}}\right)=0$ unless $n_{1} \in(\lambda)^{-3}, n_{2} \in(\lambda)^{-5}, k_{1} \equiv k_{2} \equiv 0$ or $1 \bmod (3)$, and $n_{1}^{2} n_{2}$ is a cube;

(ii) $\theta^{\rho}\left(n_{1} p^{3 k_{1}}, n_{2} p^{3 k_{2}}\right)=\mathbb{N} p^{k_{1}+k_{2}} \theta^{\rho}\left(n_{1}, n_{2}\right)$;

(iii) $\theta^{\rho}\left(n_{1} p^{3 k_{1}+1}, n_{2} p^{3 k_{2}+1}\right)=\mathbb{N} p^{k_{1}+k_{2}} \frac{\bar{g}\left(n_{1}, p\right)}{\theta^{\rho}}\left(n_{1}, n_{2}\right)$;

(iv) if $\theta^{\rho}\left(n_{1}, n_{2}\right) \neq 0$, then $g\left(n_{1}, p\right)=g\left(n_{2}, p\right)$.

This is Proposition 5.1 of [BH2], along with some other facts proved there.

If $f$ is a cubic metaplectic automorphic form on $\mathrm{GL}(3)$, another cubic metaplectic automorphic form $\hat{f}$ (the contragredient form) can be defined on GL(3) as

$$
\hat{f}\left(\tau_{3}\right)=f\left({ }^{\imath} \tau_{3}\right), \quad \text { where }{ }^{\imath} \tau_{3}=\left(\begin{array}{lll} 
& & -1 \\
-1 & &
\end{array}\right){ }^{t} \tau_{3}^{-1}\left(\begin{array}{rrr}
-1 \\
-1
\end{array}\right.
$$

This formulation will be needed in the convolution integral in Section 3. 
In addition, any cubic metaplectic automorphic form on GL(3) can be induced up to a maximal parabolic Eisenstein series on the cubic cover of GL(4). To do this, first define

$$
I\left(f, \tau_{4}, s\right)=\left(y_{1}^{3} y_{2}^{2} y_{3}\right)^{2 s} f\left(\tau_{3}\right),
$$

with $\tau_{3}$ as in (1.2). Then, if $P$ is the standard maximal parabolic subgroup of $\mathrm{GL}(4, \mathbb{C})$ consisting of matrices with bottom row $\left(\begin{array}{llll}0 & 0 & 0 & 1\end{array}\right)$, a maximal parabolic Eisenstein series is constructed by defining

$$
E\left(f, \tau_{4}, s\right)=\sum_{\gamma \in P \cap \Gamma_{4}(3) \backslash \Gamma_{4}(3)} \kappa_{4}(\gamma) I\left(f, \gamma \tau_{4}, s\right) .
$$

This converges absolutely for $\operatorname{Re}(s)$ sufficiently large and is well defined by Proposition 1.1(iv). Then $E\left(f, \tau_{4}, s\right)$ is a cubic metaplectic automorphic form on GL(4) for $\Gamma_{4}(3)$.

The last ingredient needed to formulate the Shimura type Rankin-Selberg integral is a generic cubic metaplectic cusp form on $\operatorname{GSp}(4)$.

Definition 2.4. $\phi$ is a cubic metaplectic cusp form on $\mathrm{GSp}(4)$ if

1. $\phi$ satisfies the conditions of Definition 2.1 when $\operatorname{GL}(n), \Gamma_{n}(3), \kappa_{n}, \tau_{n}$ and $\mathcal{H}_{n}$ are replaced with $\operatorname{GSp}(4), \Gamma(3), \kappa, \tau$ and $\mathcal{H}$ respectively;

2. for any cusp $\rho$ of $\Gamma_{2}(3)$,

$$
\int_{(\mathbb{C} /(3))^{3}} \phi\left(\mathcal{J}(\rho)\left(\begin{array}{cccc}
1 & & u_{3} & u_{4} \\
& 1 & u_{1} & u_{3} \\
& & 1 & \\
& & & 1
\end{array}\right) \tau\right) d u_{1} d u_{3} d u_{4}=0
$$

and

$$
\int_{(\mathbb{C} /(3))^{3}} \phi\left(\mathcal{J}(\rho)\left(\begin{array}{cccc}
1 & u_{2} & u_{3} & u_{4} \\
& 1 & & u_{3} \\
& & 1 & -u_{2} \\
& & & 1
\end{array}\right) \tau\right) d u_{2} d u_{3} d u_{4}=0 .
$$

Moreover, $\phi$ will be called a generic cubic metaplectic cusp form on GSp(4) if it also satisfies

3. There exist Whittaker-Fourier coefficients $a_{n_{1}, n_{2}}^{\rho}$, not all zero, such that if $n_{1} \in(\lambda)^{-5}, n_{1} \neq 0$ and $n_{2} \in(\lambda)^{-3}, n_{2} \neq 0$, then

$$
\begin{aligned}
\int_{\mathbb{C} /(9)} & \int_{(\mathbb{C} /(3))^{3}} \phi\left(\mathcal{J}(\rho)\left(\begin{array}{cccc}
1 & u_{2} & u_{3} & u_{4} \\
& 1 & u_{1} & u_{3}^{\prime} \\
& 1 & -u_{2} \\
& & 1
\end{array}\right) \tau\right) \\
& \times e\left(-n_{1} u_{1}-n_{2} u_{2}\right) d u_{4} d u_{3} d u_{2} d u_{1} \\
= & a_{n_{1}, n_{2}}^{\rho} \mathbb{N}\left(n_{1}^{-3 / 2} n_{2}^{-2}\right) W_{\phi}\left(\left(\begin{array}{cccc}
n_{1} n_{2} & & \\
& n_{1} & \\
& & 1 & \\
& & & n_{2}^{-1}
\end{array}\right) \tau\right),
\end{aligned}
$$

where $W_{\phi}$ is the non-degenerate Whittaker function associated to $\phi$.

See [BFrH1] for an account of the Whittaker functions mentioned in part 3 above. In the notation of [BFrH1], $W_{\phi}$ will be $W_{\nu_{1}, \nu_{2}}$, where $\nu_{1}$ and $\nu_{2}$ can be determined by the eigenvalues of $\phi$ with respect to the invariant differential operators on $\mathcal{H}$. 


\section{The Cubic GSp(4) $\times$ GL(3) CONVOLution}

Assume that $f$ is a cubic metaplectic automorphic form on GL(3) that satisfies the hypothesis of Lemma 2.2 and that $\phi$ is a cubic generic metaplectic cusp form on GSp(4). Then the main object of interest in this paper is

$$
\mathcal{I}(s, \phi)=\int_{\Gamma(3) \backslash \mathcal{H}} \overline{E(\hat{f}, \tau, \bar{s})} \phi(\tau) \frac{d y_{1} d y_{2} d x_{1} d x_{2} d x_{3} d x_{4}}{y_{1}^{7} y_{2}^{9}} .
$$

Here $d y_{i}$ and $d x_{j}$ are Lesbesgue measure on $\mathbb{R}$ and $\mathbb{C}$ respectively, and consequently the measure in the integral is the left invariant measure on $\mathcal{H}$.

The goal of this section is to prove

Theorem 3.1. With the above hypotheses on $f$ and $\phi$, (3.1) may be expressed in the form

$$
\mathcal{I}(s, \phi)=D(s) G(s)
$$

where

and

$$
D(s)=\sum_{\rho \in \Gamma_{2}(3) \backslash \Gamma_{2} / \Gamma_{2, \infty}} \sum_{\substack{n_{1} \in(\lambda)^{-5}, n_{1} \neq 0 \\ n_{2} \in(\lambda)^{-3}, n_{2} \neq 0}} \frac{a_{n_{1}, n_{2}}^{\rho} \overline{b_{n_{2}, n_{1}}^{\rho}}}{\mathbb{N}\left(n_{1} n_{2}^{2}\right)^{2 s-1 / 2}}
$$

$$
\begin{array}{r}
G(s)=\int_{0}^{\infty} \int_{0}^{\infty}\left(y_{1} y_{2}^{2}\right)^{4 s} W_{\phi}\left(\begin{array}{cccc}
y_{1} y_{2} & & & \\
& y_{1} & & \\
& & 1 & \\
& & & y_{2}^{-1}
\end{array}\right) \\
\times W_{f}\left(\begin{array}{lll}
y_{1} y_{2} & & \\
& y_{2} & \\
& & 1
\end{array}\right) \frac{d y_{1} d y_{2}}{y_{1}^{7} y_{2}^{9}} .
\end{array}
$$

Proof. Recall that a non-metplectic version of this integral has previously been considered by Bump [B2] and Gelbart and Piatetski-Shapiro [GeP-S]. As in [B2], notice that for $Q=P \cap G$, there is an identification of the coset space $\Gamma_{4}(3) \cap P \backslash \Gamma_{4}(3)$ with $\Gamma_{4}(3) \cap Q \backslash \Gamma(3)$. To see this, notice that the cosets of $P \cap \Gamma_{4}(3) \backslash \Gamma_{4}(3)$ can be parameterized by bottom rows $(A B C D)$, with $D \equiv 1 \bmod (3), A, B, C \equiv$ $0 \bmod (3)$, and $\operatorname{gcd}(A, B, C, D)=1$. Then the identification follows since any such bottom row can be extended to a matrix in $\Gamma(3)$. Friedberg [Fr] has proved this last statement by constructing the invariants necessary to apply Theorem 5.2 of [BFrH1].

Unfolding the Eisenstein series $E(\hat{f}, \tau, \bar{s})$ yields

$$
\mathcal{I}(s, \phi)=\int_{\Gamma_{4}(3) \cap Q \backslash \mathcal{H}} \overline{I(\hat{f}, \tau, \bar{s})} \phi(\tau) \frac{d y_{1} d y_{2} d x_{1} d x_{2} d x_{3} d x_{4}}{y_{1}^{7} y_{2}^{9}} .
$$

Now since $\tau \cdot y_{2}$ and $\tau$ are in the same coset of $\mathcal{H}_{4}$,

$$
I(\hat{f}, \tau, \bar{s})=I\left(\hat{f}, \tau \cdot y_{2}, \bar{s}\right)=\left(y_{1}^{2} y_{2}^{4}\right)^{2 \bar{s}} \hat{f}\left(\tau_{3}\right) .
$$

Thus $\mathcal{I}(s, \phi)$ becomes

$$
\mathcal{I}(s, \phi)=\int_{\Gamma_{4}(3) \cap Q \backslash \mathcal{H}} \phi(\tau) \overline{\hat{f}\left(\tau_{3}\right)}\left(y_{1} y_{2}^{2}\right)^{4 s} \frac{d y_{1} d y_{2} d x_{1} d x_{2} d x_{3} d x_{4}}{y_{1}^{7} y_{2}^{9}}
$$


To continue the evaluation of $\mathcal{I}(s, \phi)$, first an understanding of the quotient $\Gamma_{4}(3) \cap Q \backslash \mathcal{H}$ is required. Suppose $\gamma \in \Gamma_{2}(3)$ and $\xi=\left(\begin{array}{r}x_{3}^{\prime} \\ -x_{2}\end{array}\right)-\gamma\left(\begin{array}{r}x_{3}^{\prime} \\ -x_{2}\end{array}\right)$; then

$$
\varrho=\left(\begin{array}{ccc}
1 & & \\
& \gamma & \xi \\
& & 1
\end{array}\right) \in \Gamma_{4}(3) \cap Q
$$

and

$$
\begin{aligned}
& \varrho\left(\begin{array}{cccc}
1 & x_{2} & x_{3} & x_{4} \\
& 1 & x_{1} & x_{3}^{\prime} \\
& & 1 & -x_{2}
\end{array}\right)\left(\begin{array}{cccc}
y_{1} y_{2} & & & \\
& y_{1} & & \\
& & 1 & \\
& & & y_{2}^{-1}
\end{array}\right) \\
&=\left(\begin{array}{cccc}
1 & x_{2} & x_{3} & x_{4} \\
& \gamma\left(\begin{array}{cc}
1 & x_{1} \\
& 1
\end{array}\right) & x_{3}^{\prime} \\
& & & -x_{2} \\
& & & 1
\end{array}\right)\left(\begin{array}{cccc}
y_{1} y_{2} & & & \\
& y_{1} & & \\
& & 1 & \\
& & & y_{2}^{-1}
\end{array}\right) .
\end{aligned}
$$

Thus the range of the parameters $x_{1}, y_{1}$ in the quotient $\Gamma_{4}(3) \cap Q \backslash \mathcal{H}$ is determined by the action of $\Gamma_{2}(3)$ on $\left(\begin{array}{cc}y_{1} & x_{1} \\ & 1\end{array}\right)$. Similarly,

$$
\begin{aligned}
& \left(\begin{array}{ccccc}
1 & e & f & g \\
& & & h \\
& & \gamma & & i \\
& & & 1
\end{array}\right)\left(\begin{array}{cccc}
1 & x_{2} & x_{3} & x_{4} \\
& 1 & x_{1} & x_{3}^{\prime} \\
& & 1 & -x_{2} \\
& & & 1
\end{array}\right)\left(\begin{array}{cccc}
y_{1} y_{2} & & & \\
& y_{1} & & \\
& & 1 & \\
& & & y_{2}^{-1}
\end{array}\right) \\
& =\left(\begin{array}{cccc}
1 & x_{2}+e & x_{3}+x_{1} e+f & x_{4}+x_{3}^{\prime} e-x_{2} f+g \\
& \gamma\left(\begin{array}{cc}
1 & x_{1} \\
& 1
\end{array}\right) & \gamma\left(\begin{array}{r}
x_{3}^{\prime} \\
-x_{2}
\end{array}\right)+\left(\begin{array}{c}
h \\
i
\end{array}\right)
\end{array}\right) \\
& \times\left(\begin{array}{cccc}
y_{1} y_{2} & & & \\
& y_{1} & & \\
& & 1 & \\
& & & y_{2}^{-1}
\end{array}\right),
\end{aligned}
$$

so the range of the parameters $x_{2}, x_{3}$, and $x_{4}$ in the quotient $\Gamma_{4}(3) \cap Q \backslash \mathcal{H}$ is determined by the additive action of the ideal (3) on $\mathbb{C}$. Therefore $\Gamma_{4}(3) \cap Q \backslash \mathcal{H}$ can be realized as $\left(\Gamma_{2}(3) \backslash \mathcal{H}\right) \times(\mathbb{C} /(3))^{3} \times \mathbb{R}$.

Using this description of the fundamental domain for $\Gamma_{4}(3) \cap Q$ in (3.5) yields (3.6)

$$
\begin{aligned}
\mathcal{I}(s, \phi) & =\int_{(\mathbb{C} /(3))^{3}} \int_{0}^{\infty} \int_{\Gamma_{2}(3) \backslash \mathcal{H}_{2}} \phi(\tau) \overline{\hat{f}\left(\tau_{3}\right)}\left(y_{1} y_{2}^{2}\right)^{4 s} \frac{d x_{1} d y_{1} d y_{2} d x_{2} d x_{3} d x_{4}}{y_{1}^{7} y_{2}^{9}} \\
& =\int_{(\mathbb{C} /(3))^{2}} \int_{0}^{\infty} \int_{\Gamma_{2}(3) \backslash \mathcal{H}_{2}} \Phi(g) \overline{\hat{f}\left(\tau_{3}\right)}\left(y_{1} y_{2}^{2}\right)^{4 s} \frac{d x_{1} d y_{1} d y_{2} d x_{2} d x_{3}}{y_{1}^{7} y_{2}^{9}}
\end{aligned}
$$

where

$$
g=\left(\begin{array}{cccc}
1 & x_{2} & x_{3} & \\
& 1 & x_{1} & x_{3}^{\prime} \\
& & 1 & -x_{2} \\
& & & 1
\end{array}\right)\left(\begin{array}{cccc}
y_{1} y_{2} & & & \\
& y_{1} & & \\
& & 1 & \\
& & & y_{2}^{-1}
\end{array}\right),
$$


and

$$
\Phi(g)=\int_{\mathbb{C} /(3)} \phi\left(\left(\begin{array}{llll}
1 & & & x_{4} \\
& 1 & & \\
& & 1 & \\
& & & 1
\end{array}\right) g\right) d x_{4} .
$$

To continue further with the evaluation of this integral, Novodvorsky's idea of expressing $\Phi(g)$ in terms of the Whittaker-Fourier coefficients of $\phi$ will be employed. Note that for the quadratic GSp $(4) \times \mathrm{GL}(2)$ convolution Friedberg and Wong [FrW] have previously used this strategy, and the analysis here mirrors their work. First consider the function

$$
\Phi_{1}\left(u_{2}, u_{3} ; g\right)=\int_{\mathbb{C} /(3)} \phi\left(\left(\begin{array}{cccc}
1 & u_{2} & u_{3} & u_{4} \\
& 1 & & u_{3} \\
& & 1 & -u_{2} \\
& & & 1
\end{array}\right) g\right) d u_{4} .
$$

Since, for $n_{2}, n_{3} \in(3)$,

$$
\begin{aligned}
\Phi_{1}\left(u_{2}+n_{2}, u_{3}+n_{3} ; g\right) & =\int_{\mathbb{C} /(3)} \phi\left(\left(\begin{array}{cccc}
1 & n_{2} & n_{3} & \\
& 1 & & n_{3} \\
& & 1 & -n_{2} \\
& & & 1
\end{array}\right)\left(\begin{array}{cccc}
1 & u_{2} & u_{3} & u_{4}-n_{2} u_{3}+n_{3} u_{2} \\
& 1 & & u_{3} \\
& & 1 & -u_{2} \\
& & & 1
\end{array}\right) g\right) d u_{4} \\
= & \kappa\left(\left(\begin{array}{cccc}
1 & n_{2} & n_{3} & \\
1 & & n_{3} \\
& 1 & -n_{2}
\end{array}\right)\right) \Phi_{1}\left(u_{2}, u_{3} ; g\right) \\
= & \Phi_{1}\left(u_{2}, u_{3} ; g\right) .
\end{aligned}
$$

This last equality follows from Proposition 1.1 (iii). Thus, there exists a Fourier expansion for $\Phi_{1}$. In particular,

$$
\begin{aligned}
\Phi(g) & =\Phi_{1}(0,0 ; g) \\
& =\sum_{\substack{\alpha, \beta \in(\lambda)^{-3} \\
(\alpha, \beta) \neq(0,0)}} \int_{(\mathbb{C} /(3))^{3}} \phi\left(\left(\begin{array}{cccc}
1 & u_{2} & u_{3} & u_{4} \\
& 1 & & u_{3} \\
& & 1 & -u_{2} \\
& & & 1
\end{array}\right) g\right) e\left(-\alpha u_{2}-\beta u_{3}\right) d u_{4} d u_{3} d u_{2} .
\end{aligned}
$$

Here the cuspidal assumption on $\phi$ (Definition $2.4(2)$ ) allows the restriction that $(\alpha, \beta) \neq(0,0)$. 
Next, the sum over $\alpha, \beta \in(\lambda)^{-3}$ can be replaced by a sum over $\Gamma_{2, \infty} \backslash \Gamma_{2}$ and non-zero elements of $(\lambda)^{-3}$. Then for $\delta=\left(\begin{array}{ll}a & b \\ c & d\end{array}\right) \in \operatorname{SL}(2, \mathcal{O})$, the matrix identity

$$
\begin{aligned}
& \left(\begin{array}{cccc}
1 & u_{2} & u_{3} & u_{4} \\
& 1 & & u_{3} \\
& & 1 & -u_{2} \\
& & 1
\end{array}\right) \\
& \quad=\mathcal{J}\left(\delta^{-1}\right)\left(\begin{array}{ccccc}
1 & d u_{2}-c u_{3} & -b u_{2}+a u_{3} & \\
& 1 & & \\
& & 1 & -b u_{2}+a u_{3} \\
& & & & \\
& & & 1
\end{array}\right) \mathcal{J}(\delta)
\end{aligned}
$$

implies that

$$
\Phi(g)=\sum_{\delta \in \Gamma_{2, \infty} \backslash \Gamma_{2}} \sum_{\substack{n_{2} \in(\lambda)^{-3} \\
n_{2} \neq 0}} \int_{(\mathbb{C} /(3))^{3}} \phi\left(\mathcal{J}\left(\delta^{-1}\right)\left(\begin{array}{cccc}
1 & u_{2} & u_{3} & u_{4} \\
& 1 & & u_{3} \\
& 1 & -u_{2} \\
& & 1
\end{array}\right) \mathcal{J}(\delta) g\right)
$$

Lemma 3.2. A complete set of representatives for $\Gamma_{2, \infty} \backslash \Gamma_{2}$ is given by representatives of the form $\rho \gamma^{-1}$, where the cusp $\rho$ runs over a set of representatives for $\Gamma_{2}(3) \backslash \Gamma_{2} / \Gamma_{2, \infty}(3)$ and for each such cusp $\rho, \gamma$ runs through a set of representatives for $\rho \Gamma_{2, \infty}(3) \rho^{-1} \cap \Gamma_{2}(3) \backslash \Gamma_{2}(3)$.

Proof. Write

$$
\Gamma_{2}=\bigcup_{\rho} \Gamma_{2}(3) \rho \Gamma_{2, \infty}=\bigcup_{\rho} \Gamma_{2, \infty} \rho^{-1} \Gamma_{2}(3) .
$$

Now each right $\Gamma_{2, \infty}$ coset in $\Gamma_{2, \infty} \rho^{-1} \Gamma_{2}(3)$ can be expressed as $\Gamma_{2, \infty} \rho^{-1} \gamma$ for some $\gamma \in \Gamma_{2}(3)$. Also

$$
\Gamma_{2, \infty} \rho^{-1} \gamma=\Gamma_{2, \infty} \rho^{-1} \gamma^{\prime} \Leftrightarrow \gamma^{\prime} \gamma^{-1} \in \Gamma_{2}(3) \cap \rho \Gamma_{2, \infty} \rho^{-1} .
$$

To see this, notice that

$$
\begin{aligned}
\Gamma_{2, \infty} \rho^{-1} \gamma=\Gamma_{2, \infty} \rho^{-1} \gamma^{\prime} & \Leftrightarrow \rho^{-1} \gamma^{\prime} \in \Gamma_{2, \infty} \rho^{-1} \gamma \\
& \Leftrightarrow \gamma^{\prime} \in \rho \Gamma_{2, \infty} \rho^{-1} \gamma \\
& \Leftrightarrow \gamma^{\prime} \gamma^{-1} \in \rho \Gamma_{2, \infty} \rho^{-1}
\end{aligned}
$$

but, a priori $\gamma, \gamma^{\prime} \in \Gamma_{2}(3)$; thus,

$$
\Gamma_{2, \infty} \rho^{-1} \gamma=\Gamma_{2, \infty} \rho^{-1} \gamma^{\prime} \Leftrightarrow \gamma^{\prime} \gamma^{-1} \in \Gamma_{2}(3) \cap \rho \Gamma_{2, \infty} \rho^{-1}
$$

So

$$
\Gamma_{2, \infty} \rho^{-1} \Gamma_{2}(3)=\bigcup_{\gamma \in \Gamma_{2}(3) \cap \rho \Gamma_{2, \infty} \rho^{-1} \backslash \Gamma_{2}(3)} \Gamma_{2, \infty} \rho^{-1} \gamma
$$

But since $\Gamma_{2}(3) \unlhd \Gamma_{2}$, then $\Gamma_{2}(3) \cap \rho \Gamma_{2, \infty} \rho^{-1}=\rho \Gamma_{2, \infty}(3) \rho^{-1}$. So doing this for each $\rho$ yields the lemma. 
Now Lemma 3.2 and Proposition 1.1 imply

$$
\begin{aligned}
\Phi(g)= & \sum_{\rho \in \Gamma_{2}(3) \backslash \Gamma_{2} / \Gamma_{2, \infty}} \sum_{\substack{n_{2} \in(\lambda)^{-3} \\
n_{2} \neq 0}} \sum_{\gamma \in \rho \Gamma_{2, \infty}(3) \rho^{-1} \backslash \Gamma_{2}(3)} \kappa_{2}(\gamma) \\
& \times \int_{(\mathbb{C} /(3))^{3}} \phi\left(\mathcal{J}(\rho)\left(\begin{array}{cccc}
1 & u_{2} & u_{3} & u_{4} \\
& 1 & & u_{3} \\
& & 1 & -u_{2} \\
& & & 1
\end{array}\right) \mathcal{J}\left(\rho^{-1} \gamma\right) g\right) \\
& \times e\left(-n_{2} u_{2}\right) d u_{4} d u_{3} d u_{2} .
\end{aligned}
$$

Next, another Fourier expansion will be used to continue the evaluation of $\Phi(g)$. Consider

$$
\begin{aligned}
\Phi_{2}\left(u_{1} ; g\right)= & \sum_{\rho \in \Gamma_{2}(3) \backslash \Gamma_{2} / \Gamma_{2, \infty}} \sum_{\substack{n_{2} \in(\lambda)^{-3} \\
n_{2} \neq 0}} \sum_{\gamma \in \rho \Gamma_{2, \infty}(3) \rho^{-1} \backslash \Gamma_{2}(3)} \kappa_{2}(\gamma) \\
& \times \int_{(\mathbb{C} /(3))^{3}} \phi\left(\mathcal{J}(\rho)\left(\begin{array}{cccc}
1 & u_{2} & u_{3} & u_{4} \\
& 1 & u_{1} & u_{3}^{\prime} \\
& & 1 & -u_{2} \\
& & & 1
\end{array}\right) \mathcal{J}\left(\rho^{-1} \gamma\right) g\right) \\
& \times e\left(-n_{2} u_{2}\right) d u_{4} d u_{3} d u_{2},
\end{aligned}
$$

where $u_{3}^{\prime}=u_{3}-u_{1} u_{2}$. This $\Phi_{2}$ was obtained from (3.8) by changing the matrix that depends on $u_{2}, u_{3}$, and $u_{4}$ to a symplectic matrix also depending on $u_{1}$.

Now since

$$
\begin{gathered}
\left(\begin{array}{cccc}
1 & & & \\
& 1 & n_{1} & \\
& & 1 & \\
& & 1
\end{array}\right)\left(\begin{array}{cccc}
1 & u_{2} & u_{3} & u_{4} \\
& 1 & u_{1} & u_{3}^{\prime} \\
& & 1 & -u_{2} \\
& & 1
\end{array}\right) \\
=\left(\begin{array}{cccc}
1 & u_{2} & u_{3} & u_{4} \\
& 1 & u_{1}+n_{1} & u_{3}-\left(u_{1}+n_{1}\right) u_{2} \\
& & 1 & -u_{2} \\
& & &
\end{array}\right)
\end{gathered}
$$

it follows from a change of variables that

$$
\Phi_{2}\left(u_{1}+n_{1} ; g\right)=\overline{\kappa\left(\mathcal{J}(\rho) \mathcal{J}\left(\begin{array}{cc}
1 & n_{1} \\
& 1
\end{array}\right) \mathcal{J}(\rho)^{-1}\right)} \Phi_{2}\left(u_{1} ; g\right) .
$$


So making the assumption that $n_{1} \in(9)$, Lemma 1.2 implies that $\Phi_{2}\left(u_{1} ; g\right)$ has a Fourier expansion. In particular,

$$
\begin{aligned}
\Phi(g) & =\Phi_{2}(0 ; g)=\sum_{n_{1} \in(\lambda)^{-5}} \int_{\mathbb{C} /(9)} \Phi_{2}\left(u_{1} ; g\right) e\left(-n_{1} u_{1}\right) d u_{1} \\
& =\sum_{\rho \in \Gamma_{2}(3) \backslash \Gamma_{2} / \Gamma_{2, \infty}} \sum_{\substack{n_{2} \in(\lambda)^{-3}, n_{2} \neq 0 \\
n_{1} \in(\lambda)^{-5}, n_{1} \neq 0}} \sum_{2 \in \rho \Gamma_{2, \infty}(3) \rho^{-1} \backslash \Gamma_{2}(3)} \kappa_{2} \\
& \times \int_{\mathbb{C} /(9)} \int_{(\mathbb{C} /(3))^{3}} \phi\left(\mathcal{J}(\rho)\left(\begin{array}{cccc}
1 & u_{2} & u_{3} & u_{4} \\
& 1 & u_{1} & u_{3}^{\prime} \\
& & 1 & -u_{2} \\
& & & 1
\end{array}\right) \mathcal{J}\left(\rho^{-1} \gamma\right) g\right) \\
& \times e\left(-n_{1} u_{1}-n_{2} u_{2}\right) d u_{4} d u_{3} d u_{2} d u_{1} .
\end{aligned}
$$

Here the cuspidal assumption on $\phi$ (Definition $2.4(2))$ has allowed the restriction that $n_{1} \neq 0$.

Now replace the expression of $\Phi(g)$ in (3.6) with (3.9), use (2.4) from the hypothesis that $\phi$ is generic, and write $\overline{\hat{f}\left(\tau_{3}\right)}=\overline{\kappa_{3}\left(\begin{array}{cc}1 & \\ & \gamma\end{array}\right) \hat{f}\left(\left(\begin{array}{cc}1 & \\ & \gamma\end{array}\right) \tau_{3}\right)}$ for $\gamma \in \Gamma_{2}(3)$. Then

$$
\begin{aligned}
& \mathcal{I}(s, \phi)=\int_{(\mathbb{C} /(3))^{2}} \int_{0}^{\infty} \sum_{\rho \in \Gamma_{2}(3) \backslash \Gamma_{2} / \Gamma_{2, \infty}} \sum_{\substack{n_{1} \in(\lambda)^{-5}, n_{1} \neq 0 \\
n_{2} \in(\lambda)^{-3}, n_{2} \neq 0}} a_{n_{1}, n_{2}}^{\rho} \mathbb{N} n_{1}^{-3 / 2} \mathbb{N} n_{2}^{-2} \\
& \times \sum_{\gamma \in \rho \Gamma_{2, \infty}(3) \rho^{-1} \cap \Gamma_{2}(3) \backslash \Gamma_{2}(3)} \kappa_{2}(\gamma) \overline{\kappa_{3}\left(\begin{array}{cc}
1 & \\
& \gamma
\end{array}\right)} \\
& \times \int_{\Gamma_{2}(3) \backslash \mathcal{H}_{2}} W_{\phi}\left(\left(\begin{array}{cccc}
n_{1} n_{2} & & & \\
& n_{1} & & \\
& & 1 & \\
& & & n_{2}^{-1}
\end{array}\right) \mathcal{J}\left(\rho^{-1} \gamma\right) g\right) \\
& \times \overline{\hat{f}\left(\left(\begin{array}{ll}
1 & \\
& \gamma
\end{array}\right) \tau_{3}\right)}\left(y_{1} y_{2}^{2}\right)^{4 s} \frac{d x_{1} d y_{1} d y_{2} d x_{2} d x_{3}}{y_{1}^{7} y_{2}^{9}} \text {. }
\end{aligned}
$$

Write $\overline{\kappa_{3}\left(\begin{array}{cc}1 & \\ & \gamma\end{array}\right)}=\kappa_{2}(\gamma)^{-1}$ and sum over $\gamma$ to collapse the integral further. Also the change of variables $g \mapsto \mathcal{J}(\rho) g$ forces $\tau_{3} \mapsto\left(\begin{array}{cc}1 & \\ & \rho\end{array}\right) \tau_{3}$. Then, since the action 
of $\rho$ preserves fundamental domains of $\Gamma_{2, \infty}(3)$,

$$
\begin{aligned}
& \mathcal{I}(s, \phi)=\sum_{\rho \in \Gamma_{2}(3) \backslash \Gamma_{2} / \Gamma_{2, \infty}} \sum_{\substack{n_{1} \in(\lambda)^{-5}, n_{1} \neq 0 \\
n_{2} \in(\lambda)^{-3}, n_{2} \neq 0}} a_{n_{1}, n_{2}}^{\rho} \mathbb{N} n_{1}^{-3 / 2} \mathbb{N} n_{2}^{-2} \int_{0}^{\infty} \int_{0}^{\infty} \\
& \times\left[\int_{(\mathbb{C} /(3))^{3}} \overline{\hat{f}\left(\left(\begin{array}{cc}
1 & \\
& \rho
\end{array}\right) \tau_{3}\right)} e\left(n_{1} x_{1}+n_{2} x_{2}\right) d x_{1} d x_{2} d x_{3}\right] \\
& \times W_{\phi}\left(\left(\begin{array}{cccc}
n_{1} n_{2} & & & \\
& n_{1} & & \\
& & 1 & \\
& & & n_{2}^{-1}
\end{array}\right)\left(\begin{array}{llll}
y_{1} y_{2} & & & \\
& y_{1} & & \\
& & 1 & \\
& & & y_{2}^{-1}
\end{array}\right)\right) \\
& \times\left(y_{1} y_{2}^{2}\right)^{4 s} \frac{d y_{1} d y_{2}}{y_{1}^{7} y_{2}^{9}} \text {. }
\end{aligned}
$$

Here the equality

$$
\begin{aligned}
& W_{\phi}\left(\left(\begin{array}{cccc}
n_{1} n_{2} & & & \\
& n_{1} & & \\
& & 1 & \\
& & n_{2}^{-1}
\end{array}\right) g\right) \\
& =e\left(n_{1} x_{1}+n_{2} x_{2}\right) W_{\phi}\left(\left(\begin{array}{llll}
n_{1} n_{2} y_{1} y_{2} & & \\
& n_{1} y_{1} & \\
& & 1 & \\
& & & n_{2}^{-1} y_{2}^{-1}
\end{array}\right)\right)
\end{aligned}
$$

has been used. It may be proved by using a change of variables in (2.4).

Now the integral in brackets in (3.10) is expressible in terms of a WhittakerFourier coefficient of $f$. To see this notice that

$$
\begin{aligned}
\int_{(\mathbb{C} /(3))^{3}} & \overline{\hat{f}\left(\left(\begin{array}{cc}
1 & \\
& \rho
\end{array}\right) \tau_{3}\right)} e\left(n_{1} x_{1}+n_{2} x_{2}\right) d x_{1} d x_{2} d x_{3} \\
\quad= & \int_{(\mathbb{C} /(3))^{3}} \hat{f}\left(\left(\begin{array}{ll}
\rho^{*} & \\
& 1
\end{array}\right)\left(\begin{array}{ccc}
1 & -x_{1} & x_{1} x_{2}-x_{3} \\
1 & -x_{2} \\
& 1
\end{array}\right)\left(\begin{array}{ccc}
y_{1} y_{2} & \\
& y_{2} & \\
& & 1
\end{array}\right)\right) \\
& \times e\left(n_{1} x_{1}+n_{2} x_{2}\right) d x_{1} d x_{2} d x_{3} .
\end{aligned}
$$

So by changing variables and using (2.1) this integral becomes

$$
\overline{b_{-n_{2},-n_{1}}^{\rho^{*}}} \mathbb{N}\left(n_{1} n_{2}\right)^{-1} W_{f}\left(\left(\begin{array}{ccc}
\left(-n_{1}\right)\left(-n_{2}\right) & & \\
& -n_{2} & \\
& & 1
\end{array}\right)\left(\begin{array}{ccc}
y_{1} y_{2} & & \\
& y_{2} & \\
& & 1
\end{array}\right)\right) .
$$

But $W_{f}$ is a function on $\mathcal{H}_{3}$, so the matrix identity

$$
\begin{aligned}
& \left(\begin{array}{ccc}
\left(-n_{1}\right)\left(-n_{2}\right) & & \\
& -n_{2} & \\
& & 1
\end{array}\right)\left(\begin{array}{ccc}
y_{1} y_{2} & & \\
& y_{2} & \\
& & 1
\end{array}\right) \\
& \quad=\left(\begin{array}{lll}
n_{1} n_{2} & & \\
& n_{2} & \\
& & 1
\end{array}\right)\left(\begin{array}{lll}
y_{1} y_{2} & & \\
& y_{2} & \\
& & 1
\end{array}\right)\left(\begin{array}{ccc}
1 & & \\
& -1 & \\
& & 1
\end{array}\right)
\end{aligned}
$$


and Lemma 2.2 imply that the integral in brackets in (3.10) is just

$$
\overline{b_{n_{2}, n_{1}}^{\rho}} \mathbb{N}\left(n_{1} n_{2}\right)^{-1} W_{f}\left(\left(\begin{array}{lll}
n_{1} n_{2} & & \\
& n_{2} & \\
& & 1
\end{array}\right)\left(\begin{array}{lll}
y_{1} y_{2} & & \\
& y_{2} & \\
& & 1
\end{array}\right)\right) .
$$

Thus by integrating $x_{2}$ over $\mathbb{C} /(9)$ in (3.10), we see that there exist constants $b_{n_{2}, n_{1}}^{\rho}$ (via $(3.11)$ ) such that

$$
\begin{aligned}
& \mathcal{I}(s, \phi)=\sum_{\rho \in \Gamma_{2}(3) \backslash \Gamma_{2} / \Gamma_{2, \infty}} \sum_{\substack{n_{1} \in(\lambda)^{-5}, n_{1} \neq 0 \\
n_{2} \in(\lambda)^{-3}, n_{2} \neq 0}} a_{n_{1}, n_{2}}^{\rho} \overline{b_{n_{2}, n_{1}}^{\rho}} \mathbb{N} n_{1}^{-5 / 2} \mathbb{N} n_{2}^{-3} \\
& \times \int_{0}^{\infty} \int_{0}^{\infty} W_{\phi}\left(\left(\begin{array}{cccc}
n_{1} n_{2} & & & \\
& n_{1} & & \\
& & 1 & \\
& & & n_{2}^{-1}
\end{array}\right)\left(\begin{array}{cccc}
y_{1} y_{2} & & & \\
& y_{1} & & \\
& & 1 & \\
& & & y_{2}^{-1}
\end{array}\right)\right) \\
& \times \overline{W_{f}\left(\left(\begin{array}{lll}
n_{1} n_{2} & & \\
& n_{2} & \\
& & 1
\end{array}\right)\left(\begin{array}{lll}
y_{1} y_{2} & & \\
& y_{2} & \\
& & 1
\end{array}\right)\right)}\left(y_{1} y_{2}^{2}\right)^{4 s} \frac{d y_{1} d y_{2}}{y_{1}^{7} y_{2}^{9}} .
\end{aligned}
$$

The change of variables $y_{1} \mapsto \frac{y_{1}}{\left|n_{1}\right|}$ and $y_{2} \mapsto \frac{y_{2}}{\left|n_{2}\right|}$ then yields (3.2), and hence completes the proof of Theorem 3.1.

\section{Cubic metaplectic Hecke operators}

The fact that $D(s)$ has an expression as an Euler product will be derived from the assumption that $\phi$ is an eigenfunction for almost all of the Hecke operators on the cubic cover of GSp(4). For each prime $p$ in $\mathcal{O}$, relatively prime to (3), there exist operators $T_{p^{6}}, T_{p^{3}}$ which act on the space of cubic metaplectic automorphic forms on $\mathrm{GSp}(4)$. In fact, these two operators generate the whole Hecke algebra on the cubic cover of GSp(4). The explicit action of these Hecke operators will be given by describing the effect they have on the Whittaker-Fourier coefficients of an automorphic form.

First, recall the definition of the cubic Gauss sums

$$
g(a, d)=\sum_{c \bmod d}\left(\frac{c}{d}\right)_{3} e\left(\frac{a c}{d}\right),
$$

where $d \in \mathcal{O}$ with $d \equiv 1(\bmod (3)), a \in K^{\times}$, and $c$ is selected so that $\lambda a c \in \mathcal{O}$. The primary concern here will be in the cases in which $d$ is a power of a prime. The relevant facts can be found in Proposition 1.6 of [BH3]. For clarity, they are summarized here in

Proposition 4.1. Suppose that $a$ and $p$ are coprime and $k$ is a non-negative integer. Then

(i) $g\left(a b, p^{k}\right)=\overline{\left(\frac{a}{p^{k}}\right)_{3}} g\left(b, p^{k}\right)$;

(ii) $|g(1, p)|^{2}=\mathbb{N} p$;

(iii) $g\left(p^{k}, p\right)= \begin{cases}g(1, p) & \text { if } k=0 \\ 0 & \text { otherwise; }\end{cases}$ 
(iv) $g\left(p^{k}, p^{2}\right)= \begin{cases}\mathbb{N} p \overline{g(1, p)} & \text { if } k=1 \\ 0 & \text { otherwise. }\end{cases}$

To define the Hecke operators, first recall that every prime ideal coprime with (3) has a unique generator $p \equiv 1 \bmod (3)$. Now suppose

$$
\xi_{p^{6}}=\left(\begin{array}{cccc}
p^{6} & & & \\
& p^{3} & & \\
& & p^{3} & \\
& & & 1
\end{array}\right) \text { and } \xi_{p^{3}}=\left(\begin{array}{cccc}
p^{3} & & & \\
& p^{3} & & \\
& & 1 & \\
& & & 1
\end{array}\right) \text {. }
$$

For $\eta=\xi_{p^{6}}$ or $\xi_{p^{3}}, \Gamma(3) \eta \Gamma(3)$ can be written as a disjoint union of right cosets

$$
\Gamma(3) \eta \Gamma(3)=\bigcup_{i=1}^{m} \Gamma(3) \eta_{i}, \quad \eta_{i}=\gamma_{i} \eta \delta_{i} \quad \gamma_{i}, \delta_{i} \in \Gamma(3) .
$$

Then $T_{\eta}$ acts on the space of cubic metaplectic automorphic forms on GSp(4) by

$$
\left(T_{\eta} \cdot \phi\right)(\tau)=\sum_{i=1}^{m} \kappa\left(\gamma_{i}\right) \kappa\left(\delta_{i}\right) \phi\left(\eta_{i} \tau\right) .
$$

In what follows write $T_{p^{6}}$ and $T_{p^{3}}$ for $T_{\xi_{p^{6}}}$ and $T_{\xi_{p^{3}}}$ respectively.

As the Whittaker-Fourier coefficients in (2.4) are parameterized by the cusps $\rho$ (see (1.4)), there is a need to understand an action of $K^{\times}$on the cusps. As in Bump and Hoffstein [BH2], for $d \in K^{\times}$with numerator and denominator (written in reduced form) coprime with 3 , define $d[\rho]$ to be the $\operatorname{coset}\left(\begin{array}{ll}d & \\ & 1\end{array}\right) \rho\left(\begin{array}{ll}d & \\ & 1\end{array}\right)^{-1}$. Here representatives $\rho$ are taken such that $\rho, d[\rho] \in \Gamma_{2}$.

Theorem 4.2. If $a^{\rho}\left(n_{1}, n_{2}\right), b^{\rho}\left(n_{1}, n_{2}\right)$, and $c^{\rho}\left(n_{1}, n_{2}\right)$ are the Whittaker-Fourier coefficients of $\phi, T_{p^{6}} \cdot \phi$, and $T_{p^{3}} \cdot \phi$ respectively, then

$$
\begin{aligned}
& b^{\rho}\left(n_{1}, n_{2}\right) \\
& =\mathbb{N} p^{6}\left[a^{\rho}\left(n_{1}, n_{2} p^{-3}\right)+a^{p^{6}[\rho]}\left(n_{1} p^{-6}, n_{2} p^{3}\right)\right. \\
& \left.+a^{p^{-6}[\rho]}\left(n_{1} p^{6}, n_{2} p^{-3}\right)+a^{\rho}\left(n_{1}, n_{2} p^{3}\right)\right] \\
& +\mathbb{N} p^{5}\left[a^{p^{2}[\rho]}\left(n_{1} p^{-2}, n_{2} p^{-1}\right) g\left(n_{2} p^{-1}, p\right)+a^{p^{4}[\rho]}\left(n_{1} p^{-4}, n_{2} p\right) \overline{g\left(n_{2}, p\right)}\right. \\
& +a^{p^{4}[\rho]}\left(n_{1} p^{-4}, n_{2} p^{2}\right) \overline{g\left(n_{1} p^{-4}, p\right)}+a^{p^{-4}[\rho]}\left(n_{1} p^{4}, n_{2} p^{-2}\right) g\left(n_{1}, p\right) \\
& \left.+a^{p^{-4}[\rho]}\left(n_{1} p^{4}, n_{2} p^{-1}\right) g\left(n_{2} p^{-1}, p\right)+a^{p^{-2}[\rho]}\left(n_{1} p^{2}, n_{2} p\right) \overline{g\left(n_{2}, p\right)}\right] \\
& +\mathbb{N} p^{4}\left[a^{p^{2}[\rho]}\left(n_{1} p^{-2}, n_{2}\right) \overline{g\left(n_{1} p^{-2}, p\right)} \overline{g\left(n_{2}, p\right)}\right. \\
& +a^{p^{2}[\rho]}\left(n_{1} p^{-2}, n_{2} p\right) \overline{g\left(n_{1} p^{-2}, p^{2}\right)} \\
& \left.+a^{p^{-2}[\rho]}\left(n_{1} p^{2}, n_{2} p^{-1}\right) g\left(n_{1}, p^{2}\right)+a^{p^{-2}[\rho]}\left(n_{1} p^{2}, n_{2}\right) g\left(n_{1}, p\right) g\left(n_{2}, p\right)\right] \\
& +a^{\rho}\left(n_{1}, n_{2}\right) \begin{cases}-\mathbb{N} p^{4} & \text { if }\left(p \nmid n_{1} n_{2}\right) \text { or }\left(p^{2} \mid n_{1} \text { and } p^{3} \nmid n_{1}\right), \\
\mathbb{N} p^{4}(\mathbb{N} p-1) & \text { if }\left(p \mid n_{2} \text { and } p^{2} \nmid n_{1}\right), \\
\mathbb{N} p^{4}(\mathbb{N} p-1) & \text { if }\left(p \mid n_{1} \text { and } p^{2} \nmid n_{1}\right), \\
\mathbb{N} p^{4}\left(\mathbb{N} p^{2}-1\right) & \text { if } p^{3} \mid n_{1},\end{cases}
\end{aligned}
$$


and

$$
\begin{aligned}
& c^{\rho}\left(n_{1}, n_{2}\right) \\
& =\quad \mathbb{N}^{9 / 2}\left[a^{p^{3}[\rho]}\left(n_{1} p^{-3}, n_{2}\right)+a^{p^{-3}[\rho]}\left(n_{1} p^{3}, n_{2} p^{-3}\right)\right. \\
& \left.+a^{p^{3}[\rho]}\left(n_{1} p^{-3}, n_{2} p^{3}\right)+a^{p^{-3}[\rho]}\left(n_{1} p^{3}, n_{2}\right)\right] \\
& +\mathbb{N} p^{7 / 2}\left[a^{p[\rho]}\left(n_{1} p^{-1}, n_{2} p^{-1}\right) \overline{g\left(n_{1} p^{-1}, p\right)}+a^{p^{-1}[\rho]}\left(n_{1} p, n_{2} p^{-2}\right) g\left(n_{1}, p\right)\right. \\
& +a^{p^{-1}[\rho]}\left(n_{1} p, n_{2} p^{-1}\right) g\left(n_{2} p^{-1}, p\right)+a^{p[\rho]}\left(n_{1} p^{-1}, n_{2} p\right) \overline{g\left(n_{2}, p\right)} \\
& \left.+a^{p[\rho]}\left(n_{1} p^{-1}, n_{2} p^{2}\right) \overline{g\left(n_{1} p^{-1}, p\right)}+a^{p^{-1}[\rho]}\left(n_{1} p, n_{2} p\right) g\left(n_{1}, p\right)\right] .
\end{aligned}
$$

Here it is understood that $a_{n_{1}, n_{2}}^{\rho}=0$ unless $n_{1} \in(\lambda)^{-5}$ and $n_{2} \in(\lambda)^{-3}$. Note that for $p \equiv 1 \bmod (3), p^{k}[\rho]$ and $\rho$ are in the same double coset of $\Gamma_{2}(3) \backslash \Gamma_{2} / \Gamma_{2, \infty}$ for any $k$. Thus $a_{n_{1}, n_{2}}^{p^{k}[\rho]}=a_{n_{1}, n_{2}}^{\rho}$, and Theorem 4.2 is actually more explicit than necessary. In fact, (4.4) and (4.5) will only be used when all cusps there are the same.

Theorem 4.2 will be proved by explicitly writing down right coset representatives $\eta_{i}$ as in (4.2), computing $\kappa=\kappa\left(\gamma_{i}\right) \kappa\left(\delta_{i}\right)$, and giving the resulting contributions to the Whittaker-Fourier coefficients for both $\eta=\xi_{p^{6}}$ and $\eta=\xi_{p^{3}}$. The contribution to the Whittaker-Fourier coefficient $a_{n_{1}, n_{2}}^{\rho}$ is determined by evaluating an integral of the form

$$
\begin{gathered}
\int_{\mathbb{C} /(9)} \int_{(\mathbb{C} /(3))^{3}} \phi\left(\eta_{i} \mathcal{J}(\rho)\left(\begin{array}{cccc}
1 & u_{2} & u_{3} & u_{4} \\
& 1 & u_{1} & u_{3}^{\prime} \\
& 1 & -u_{2} \\
& & 1
\end{array}\right) \tau\right) \\
\times e\left(-n_{1} u_{1}-n_{2} u_{2}\right) d u_{4} d u_{3} d u_{2} d u_{1} .
\end{gathered}
$$

To do this, write

$$
\eta_{i} \mathcal{J}(\rho)=\eta_{i} \mathcal{J}(\rho) \eta_{i}^{-1} \eta_{i}=\mathcal{J}(d[\rho]) \eta_{i}
$$

for some $d$. Then the contribution is determined by changing variables in $u_{i}$ to obtain an integral in the form of (2.4).

For the operator $T_{p^{6}}$, the coset representatives are:

$$
\text { I. }\left(\begin{array}{cccc}
p^{6} & & & \\
& p^{3} & & \\
& & p^{3} & \\
& & & 1
\end{array}\right),\{\kappa=1\} \text {. }
$$

This coset contributes $\mathbb{N} p^{6} a^{\rho}\left(n_{1}, n_{2} p^{-3}\right)$.

$$
\text { II. }\left(\begin{array}{cccc}
p^{5} & a p^{3} & & \\
& p^{4} & & \\
& & p^{2} & -a \\
& & & p
\end{array}\right),\left\{a \bmod p, p \nmid a, \kappa=\left(\frac{a}{p}\right)\right\} \text {. }
$$

These cosets contribute $\mathbb{N} p^{5} a^{p^{2}[\rho]}\left(n_{1} p^{-2}, n_{2} p^{-1}\right) g\left(n_{2} p^{-1}, p\right)$. 
III. $\left(\begin{array}{cccc}p^{5} & & e p^{2} & d \\ & p^{3} & b p^{2} & e \\ & & p^{3} & \\ & & & p\end{array}\right),\left\{\begin{array}{l}b, d, e \bmod p, p \nmid d, \\ e^{2} \equiv b d \bmod p, \kappa=\left(\frac{d}{p}\right)^{-1}\end{array}\right\}$.

These cosets contribute zero.

$$
\mathrm{IV} .\left(\begin{array}{cccc}
p^{5} & & e p^{3} & \\
& p^{2} & b p^{2} & e \\
& & p^{4} & \\
& & & p
\end{array}\right),\left\{e \bmod p, b \bmod p^{2}, p \nmid e, \kappa=\left(\frac{e}{p}\right)\right\} .
$$

These cosets contribute zero.

$$
\mathrm{V} .\left(\begin{array}{cccc}
p^{4} & a p^{3} & & \\
& p^{5} & & \\
& & p & -a \\
& & & p^{2}
\end{array}\right),\left\{a \bmod p^{2}, p \nmid a, \kappa=\left(\frac{a}{p}\right)^{-1}\right\} .
$$

These cosets contribute $\mathbb{N} p^{5} a^{p^{4}[\rho]}\left(n_{1} p^{-4}, n_{2} p\right) \overline{g\left(n_{2}, p\right)}$.

$$
\text { VI. }\left(\begin{array}{cccc}
p^{4} & a p^{3} & e+a b & d \\
& p^{4} & b p & e \\
& & p^{2} & -a p \\
& & & p^{2}
\end{array}\right),\left\{\begin{array}{l}
a, b \bmod p, e, d \bmod p^{2} \\
e(a b+e) \equiv b d p \bmod p^{3} \\
a(a b+e) \equiv-d p \bmod p^{2} \\
p \nmid a b e, \kappa=\left(\frac{a b}{p}\right)^{-1}
\end{array}\right\} \text {. }
$$

These cosets contribute $\mathbb{N} p^{4} a^{p^{2}[\rho]}\left(n_{1} p^{-2}, n_{2}\right) \overline{g\left(n_{1} p^{-2}, p\right)} \overline{g\left(n_{2}, p\right)}$.

$$
\text { VII. }\left\{\begin{array}{cccc}
p^{4} & & e p & d \\
& p^{3} & b p & e \\
& & p^{3} & \\
& & & p^{2}
\end{array}\right),\left\{\begin{array}{l}
b, d, e \bmod p^{2}, p \nmid d, \\
e^{2} \equiv b d \bmod p, \kappa=\left(\frac{d}{p}\right)
\end{array}\right\} .
$$

These cosets contribute zero.

$$
\text { VIII. }\left\{\begin{array}{cccc}
p^{4} & a p^{2} & e p^{2} & d \\
& p^{3} & & e p \\
& & p^{3} & -a p \\
& & & p^{2}
\end{array}\right),\left\{\begin{array}{l}
a, e \bmod p, d \bmod p^{2} \\
p \nmid a d, \kappa=\left(\frac{d}{p}\right)
\end{array}\right\} .
$$

These cosets contribute zero.

$$
\operatorname{IX.}\left(\begin{array}{cccc}
p^{4} & & e p^{2} & d p \\
& p^{2} & b p & e \\
& & p^{4} & \\
& & & p^{2}
\end{array}\right),\left\{\begin{array}{l}
d \bmod p, e \bmod p^{2}, b \bmod p^{3}, p \nmid b d e, \\
e^{2} \equiv b d \bmod p, \kappa=\left(\frac{b e^{2}}{p}\right)
\end{array}\right\} .
$$

These cosets contribute zero.

$$
\mathrm{X} .\left(\begin{array}{cccc}
p^{4} & & e p^{3} & \\
& p & b p & e \\
& & p^{5} & \\
& & & p^{2}
\end{array}\right),\left\{e \bmod p^{2}, b \bmod p^{4}, p \nmid e, \kappa=\left(\frac{e}{p}\right)^{-1}\right\} \text {. }
$$

These cosets contribute zero. 
XI. $\left(\begin{array}{cccc}p^{3} & a p^{3} & & \\ & p^{6} & & \\ & & 1 & -a \\ & & & p^{3}\end{array}\right),\left\{a \bmod p^{3}, \kappa=1\right\}$.

These cosets contribute $\mathbb{N} p^{6} a^{p^{6}[\rho]}\left(n_{1} p^{-6}, n_{2} p^{3}\right)$.

$$
\text { XII. }\left\{\begin{array}{cccc}
p^{3} & a p^{3} & c & d \\
& p^{5} & b & c p^{2}-a b \\
& & p & -a p \\
& & & p^{3}
\end{array}\right),\left\{\begin{array}{l}
b, c \bmod p, a \bmod p^{2}, d \bmod p^{3}, \\
b(a c+d) \equiv c^{2} p^{2} \bmod p^{3}, \\
p \nmid b, \kappa=\left(\frac{b}{p}\right)^{-1}
\end{array}\right\} \text {. }
$$

These cosets contribute $\mathbb{N} p^{5} a^{p^{4}[\rho]}\left(n_{1} p^{-4}, n_{2} p^{2}\right) \overline{g\left(n_{1} p^{-4}, p\right)}$.

$$
\text { XIII. }\left\{\begin{array}{cccc}
p^{3} & a p^{3} & c & d \\
& p^{4} & b & c p-a b \\
& & p^{2} & -a p^{2} \\
& & & p^{3}
\end{array}\right),\left\{\begin{array}{l}
a \bmod p, b, c \bmod p^{2}, d \bmod p^{3}, \\
b(a c+d) \equiv c^{2} p \bmod p^{3}, \\
p \nmid a b, \kappa=\left(\frac{b}{p^{2}}\right)^{-1}
\end{array}\right\} \text {. }
$$

These cosets contribute $\mathbb{N} p^{3}(\mathbb{N} p-1) a^{p^{2}[\rho]}\left(n_{1} p^{-2}, n_{2} p\right) \overline{g\left(n_{1} p^{-2}, p^{2}\right)}$.

$$
\text { XIV. }\left\{\begin{array}{cccc}
p^{3} & & c & d p \\
& p^{4} & b & c p \\
& & p^{2} & \\
& & & p^{3}
\end{array}\right),\left\{\begin{array}{l}
b, c, d \bmod p^{2}, p \nmid b, \\
c^{2} \equiv b d \bmod p^{2}, \kappa=\left(\frac{b}{p^{2}}\right)^{-1}
\end{array}\right\} \text {. }
$$

These cosets contribute $\mathbb{N} p^{3} a^{p^{2}[\rho]}\left(n_{1} p^{-2}, n_{2} p\right) \overline{g\left(n_{1} p^{-2}, p^{2}\right)}$.

$$
\mathrm{XV} .\left(\begin{array}{cccc}
p^{3} & & e & d \\
& p^{3} & b & e \\
& & p^{3} & \\
& & & p^{3}
\end{array}\right),\left\{\begin{array}{l}
b, d, e \bmod p^{3}, p \nmid b, \\
e^{2} \equiv b d \bmod p^{3}, \kappa=1
\end{array}\right\} .
$$

These cosets contribute $\left\{\begin{array}{ll|l}\mathbb{N} p^{5}(\mathbb{N} p-1) a^{\rho}\left(n_{1}, n_{2}\right) & \text { if } p^{3} \mid n_{1}, \\ -\mathbb{N} p^{5} a^{\rho}\left(n_{1}, n_{2}\right) & \text { if } p^{2} \mid n_{1}, \text { but } p^{3} \nmid n_{1}, \\ 0 & \text { if } p^{2} \nmid n_{1} .\end{array}\right.$

$$
\text { XVI. }\left\{\begin{array}{cccc}
p^{3} & & e p & d \\
& p^{3} & b p^{2} & e p \\
& & p^{3} & \\
& & & p^{3}
\end{array}\right),\left\{\begin{array}{l}
b \bmod p, e \bmod p^{2}, d \bmod p^{3}, p \nmid d, \\
e^{2} \equiv b d \bmod p=1
\end{array}\right\} \text {. }
$$

These cosets contribute $\begin{cases}\mathbb{N} p^{4}(\mathbb{N} p-1) a^{\rho}\left(n_{1}, n_{2}\right) & \text { if } p \mid n_{1}, \\ 0 & \text { if } p \nmid n_{1} .\end{cases}$

$$
\text { XVII. }\left(\begin{array}{cccc}
p^{3} & a p^{2} & e p^{2} & d \\
& p^{3} & & e p^{2} \\
& & p^{3} & -a p^{2} \\
& & & p^{3}
\end{array}\right),\left\{a, e \bmod p, d \bmod p^{3}, p \nmid a d, \kappa=1\right\} \text {. }
$$

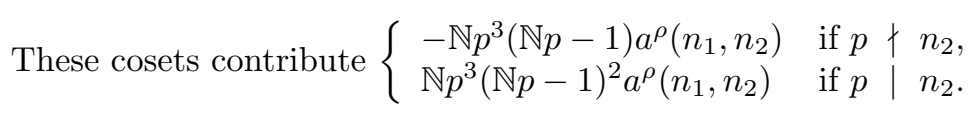




$$
\text { XVIII. }\left\{\begin{array}{cccc}
p^{3} & a p^{2} & e p+a b p & d \\
& p^{3} & b p^{2} & e p \\
& & p^{3} & -a p^{2} \\
& & & p^{3}
\end{array}\right),\left\{\begin{array}{l}
a, b \bmod p, e \bmod p^{2}, d \bmod p^{3}, \\
e(a b+e) \equiv b d \bmod p, \\
p \nmid a b d e, \kappa=\left(\frac{b d e}{p}\right)
\end{array}\right\} .
$$

These cosets contribute $\begin{cases}-\mathbb{N} p^{3} a^{[\rho]}\left(n_{1}, n_{2}\right) & \text { if } p \nmid n_{1} n_{2}, \\ \mathbb{N} p^{3}(\mathbb{N} p-1) a^{\rho}\left(n_{1}, n_{2}\right) & \text { if } p \mid n_{1} \text { and } p \nmid n_{2}, \\ \mathbb{N} p^{3}(\mathbb{N} p-1) a^{\rho}\left(n_{1}, n_{2}\right) & \text { if } p \nmid n_{1} \text { and } p \mid n_{2}, \\ -\mathbb{N} p^{3}(\mathbb{N} p-1)^{2} a^{\rho}\left(n_{1}, n_{2}\right) & \text { if } p \mid n_{1} \text { and } p \mid n_{2} .\end{cases}$

$$
\text { XIX. }\left\{\begin{array}{cccc}
p^{3} & & e p & d p \\
& p^{2} & b & e \\
& & p^{4} & \\
& & & p^{3}
\end{array}\right),\left\{\begin{array}{l}
d \bmod p^{2}, e \bmod p^{3}, b \bmod p^{4}, p \nmid b, \\
e^{2} \equiv b d \bmod p^{2}, \kappa=\left(\frac{b}{p^{2}}\right)
\end{array}\right\} \text {. }
$$

These cosets contribute $\mathbb{N} p^{4} a^{p^{-2}[\rho]}\left(n_{1} p^{2}, n_{2} p^{-1}\right) g\left(n_{1}, p^{2}\right)$.

$$
\mathrm{XX} .\left(\begin{array}{cccc}
p^{3} & a p & e p^{2}+a b p & d \\
& p^{2} & b p^{2} & e p \\
& & p^{4} & -a p^{2} \\
& & & p^{3}
\end{array}\right),\left\{\begin{array}{l}
a \bmod p, b, e \bmod p^{2}, d \bmod p^{3}, \\
p \nmid a d, p \mid a e-d, \kappa=\left(\frac{a d}{p}\right)^{-1}
\end{array}\right\} .
$$

These cosets contribute zero.

$$
\text { XXI. }\left\{\begin{array}{cccc}
p^{3} & & e p^{2} & d p^{2} \\
& p & b & e \\
& p^{5} & \\
& & & p^{3}
\end{array}\right),\left\{\begin{array}{l}
d \bmod p, e \bmod p^{3}, b \bmod p^{5}, p \nmid b, \\
e^{2} \equiv b d \bmod p, \kappa=\left(\frac{b}{p}\right)
\end{array}\right\} .
$$

These cosets contribute $\mathbb{N} p^{5} a^{p^{-4}[\rho]}\left(n_{1} p^{4}, n_{2} p^{-2}\right) g\left(n_{1}, p\right)$.

$$
\text { XXII. }\left(\begin{array}{cccc}
p^{3} & & e p^{3} & \\
& 1 & b & e \\
& & p^{6} & \\
& & & p^{3}
\end{array}\right),\left\{e \bmod p^{3}, b \bmod p^{6}, \kappa=1\right\} .
$$

These cosets contribute $\mathbb{N} p^{6} a^{p^{-6}[\rho]}\left(n_{1} p^{6}, n_{2} p^{-3}\right)$.

$$
\text { XXIII. }\left\{\begin{array}{cccc}
p^{2} & a p^{2} & c & d \\
& p^{5} & & c p^{3} \\
& & p & -a p \\
& & & p^{4}
\end{array}\right),\left\{\begin{array}{l}
c \bmod p, a \bmod p^{3}, d \bmod p^{4}, p \nmid c, \\
d \equiv a c \bmod p^{2}, \kappa=\left(\frac{c}{p}\right)
\end{array}\right\} \text {. }
$$

These cosets contribute zero.

$$
\text { XXIV. }\left(\begin{array}{cccc}
p^{2} & a p^{2} & c & d \\
& p^{4} & b p & c p^{2}-a b p \\
& & p^{2} & -a p^{2} \\
& & & p^{4}
\end{array}\right),\left\{\begin{array}{l}
b \bmod p, a, c \bmod p^{2}, d \bmod p^{4} \\
p \nmid b c, b(a c+d) \equiv c^{2} p \bmod p^{2} \\
\kappa=\left(\frac{b^{2} c}{p}\right)
\end{array}\right\} .
$$

These cosets contribute zero.

$$
\mathrm{XXV} .\left(\begin{array}{cccc}
p^{2} & a p^{2} & c & d \\
& p^{3} & b p & c p-a b p \\
& & p^{3} & -a p^{3} \\
& & & p^{4}
\end{array}\right),\left\{\begin{array}{l}
a \bmod p, b \bmod p^{2}, c \bmod p^{3} \\
d \bmod p^{4}, b(a c+d) \equiv c^{2} \bmod p^{2}, \\
p \nmid b c, \kappa=\left(\frac{b c}{p^{2}}\right)^{-1}
\end{array}\right\} .
$$

These cosets contribute zero. 


$$
\text { XXVI. }\left\{\begin{array}{cccc}
p^{2} & a p^{2} & c p & d \\
& p^{3} & & c p^{2} \\
& & p^{3} & -a p^{3} \\
& & & p^{4}
\end{array}\right),\left\{\begin{array}{l}
a \bmod p, c \bmod p^{2}, d \bmod p^{4}, \\
p \nmid c d, \kappa=\left(\frac{d}{p}\right)^{-1}
\end{array}\right\} .
$$

These cosets contribute zero.

$$
\text { XXVII. }\left\{\begin{array}{cccc}
p^{2} & a p^{2} & c p^{2} & d \\
& p^{3} & & c p^{3} \\
& & p^{3} & -a p^{3} \\
& & & p^{4}
\end{array}\right),\left\{\begin{array}{l}
a, c \bmod p, d \bmod p^{4}, \\
p \nmid d, \kappa=\left(\frac{d}{p}\right)^{-1}
\end{array}\right\} \text {. }
$$

These cosets contribute zero.

$$
\text { XXVIII. }\left(\begin{array}{cccc}
p^{2} & a p & c p & d \\
& p^{3} & & c p^{2} \\
& & p^{3} & -a p^{2} \\
& & & p^{4}
\end{array}\right),\left\{\begin{array}{l}
a, c \bmod p^{2}, d \bmod p^{4} \\
p \nmid a d, \kappa=\left(\frac{d}{p}\right)^{-1}
\end{array}\right\} .
$$

These cosets contribute zero.

$$
\text { XXIX. }\left\{\begin{array}{cccc}
p^{2} & a p & e p+a b & d \\
& p^{2} & b p & e p \\
& & p^{4} & -a p^{3} \\
& & & p^{4}
\end{array}\right),\left\{\begin{array}{l}
a \bmod p, b, e \bmod p^{3}, d \bmod p^{4}, \\
b(d-a e) \equiv e^{2} p \bmod p^{2}, \\
p \nmid a b, \kappa=\left(\frac{a b}{p}\right)
\end{array}\right\} .
$$

These cosets contribute $\mathbb{N} p^{4} a^{p^{-2}[\rho]}\left(n_{1} p^{2}, n_{2}\right) g\left(n_{1}, p\right) g\left(n_{2}, p\right)$.

$$
\text { XXX. }\left(\begin{array}{cccc}
p^{2} & a & e p^{2}+a b & d \\
& p & b p & e p \\
& & p^{5} & -a p^{3} \\
& & & p^{4}
\end{array}\right),\left\{\begin{array}{l}
a \bmod p, e \bmod p^{3}, b, d \bmod p^{4}, \\
p \nmid a, d \equiv a e \bmod p^{2}, \kappa=\left(\frac{a}{p}\right)
\end{array}\right\} .
$$

These cosets contribute $\mathbb{N} p^{5} a^{p^{-4}[\rho]}\left(n_{1} p^{4}, n_{2} p^{-1}\right) g\left(n_{2} p^{-1}, p\right)$.

$$
\text { XXXI. }\left\{\begin{array}{cccc}
p & a p & c & d \\
& p^{4} & & c p^{3} \\
& & p^{2} & -a p^{2} \\
& & & p^{5}
\end{array}\right),\left\{\begin{array}{l}
c \bmod p^{2}, a \bmod p^{3}, d \bmod p^{5}, p \nmid c, \\
p \mid a c+d, \kappa=\left(\frac{c}{p^{2}}\right)
\end{array}\right\} .
$$

These cosets contribute zero.

$$
\text { XXXII. }\left(\begin{array}{cccc}
p & a p & c p & d \\
& p^{3} & & c p^{3} \\
& & p^{3} & -a p^{3} \\
& & & p^{5}
\end{array}\right),\left\{a, c \bmod p^{2}, d \bmod p^{5}, p \nmid d, \kappa=\left(\frac{d}{p}\right)\right\} \text {. }
$$

These cosets contribute zero.

$$
\text { XXXIII. }\left\{\begin{array}{cccc}
p & a p & c & d \\
& p^{3} & b p^{2} & c p^{2}-a b p^{2} \\
& & p^{3} & -a p^{3} \\
& & & p^{5}
\end{array}\right),\left\{\begin{array}{l}
b \bmod p, a \bmod p^{2}, \\
c \bmod p^{3}, d \bmod p^{5}, \\
b(a c+d) \equiv c^{2} \bmod p, \\
p \nmid b c, \kappa=\left(\frac{b c}{p}\right)^{-1}
\end{array}\right\} \text {. }
$$

These cosets contribute zero.

$$
\text { XXXIV. }\left(\begin{array}{cccc}
p & a & c & d \\
& p^{2} & b p^{2} & c p-a b p \\
& p^{4} & -a p^{3} \\
& & & p^{5}
\end{array}\right),\left\{\begin{array}{l}
a, b \bmod p^{2}, c \bmod p^{4}, d \bmod p^{5}, \\
p \nmid a, p^{2} \mid\left(a^{2} b-a c+d p\right), \\
\kappa=\left(\frac{a}{p}\right)^{-1}
\end{array}\right\} \text {. }
$$

These cosets contribute $\mathbb{N} p^{5} a^{p^{-2}[\rho]}\left(n_{1} p^{2}, n_{2} p\right) \overline{g\left(n_{2}, p\right)}$. 


$$
\text { XXXV. }\left(\begin{array}{cccc}
1 & a & c & d \\
& p^{3} & & c p^{3} \\
& & p^{3} & -a p^{3} \\
& & & p^{6}
\end{array}\right),\left\{a, c \bmod p^{3}, d \bmod p^{6}, \kappa=1\right\} .
$$

These cosets contribute $\mathbb{N} p^{6} a^{\rho}\left(n_{1}, n_{2} p^{3}\right)$.

Since $p \equiv 1 \bmod (3)$, choose $a, b, c, d$, and $e$ above (and below) from the ideal (3). This is necessary to ensure that $\kappa\left(\delta_{i}\right)$ and $\kappa\left(\gamma_{i}\right)$ are defined.

For the operator $T_{p^{3}}$, the representatives are:

$$
\mathrm{I}^{\prime} \cdot\left(\begin{array}{cccc}
p^{3} & & & \\
& p^{3} & & \\
& & 1 & \\
& & & 1
\end{array}\right),\{\kappa=1\} \text {. }
$$

This coset contributes $\mathbb{N} p^{9 / 2} a^{p^{3}[\rho]}\left(n_{1} p^{-3}, n_{2}\right)$.

$$
\mathrm{II}^{\prime} .\left(\begin{array}{cccc}
p^{3} & & & \\
& p^{2} & b & \\
& & p & \\
& & & 1
\end{array}\right),\left\{b \bmod p, p \nmid b, \kappa=\left(\frac{b}{p}\right)^{-1}\right\} .
$$

These cosets contribute $\mathbb{N} p^{7 / 2} a^{p[\rho]}\left(n_{1} p^{-1}, n_{2} p^{-1}\right) \overline{g\left(n_{1} p^{-1}, p\right)}$.

$$
\text { III'. }\left(\begin{array}{cccc}
p^{3} & & & \\
& p & b & \\
& & p^{2} & \\
& & & 1
\end{array}\right),\left\{b \bmod p^{2}, p \nmid b, \kappa=\left(\frac{b}{p}\right)\right\} .
$$

These cosets contribute $\mathbb{N} p^{7 / 2} a^{p^{-1}[\rho]}\left(n_{1} p, n_{2} p^{-2}\right) g\left(n_{1}, p\right)$.

$$
\mathrm{IV}^{\prime} .\left(\begin{array}{cccc}
p^{3} & & & \\
& 1 & b & \\
& & p^{3} & \\
& & & 1
\end{array}\right),\left\{b \bmod p^{3}, \kappa=1\right\} .
$$

These cosets contribute $\mathbb{N} p^{9 / 2} a^{p^{-3}[\rho]}\left(n_{1} p^{3}, n_{2} p^{-3}\right)$.

$$
\mathrm{V}^{\prime} \cdot\left(\begin{array}{cccc}
p^{2} & a p^{2} & & d \\
& p^{3} & & \\
& & 1 & -a \\
& & p
\end{array}\right),\left\{a, d \bmod p, p \nmid d, \kappa=\left(\frac{d}{p}\right)^{-1}\right\} .
$$

These cosets contribute zero.

$$
\mathrm{VI}^{\prime} .\left(\begin{array}{cccc}
p^{2} & a p & c & d \\
& p^{2} & & c \\
& & p & -a \\
& & & p
\end{array}\right),\left\{a, c, d \bmod p, p \nmid c, \kappa=\left(\frac{c}{p}\right)\right\} .
$$

These cosets contribute zero.

$$
\mathrm{VII}^{\prime} .\left(\begin{array}{llll}
p^{2} & & c & d \\
& p^{2} & b & c \\
& & p & \\
& & & p
\end{array}\right),\left\{\begin{array}{l}
b, c, d \bmod p, p \nmid b\left(c^{2}-b d\right), \\
\kappa=\left(\frac{c^{2}-b d}{p}\right)^{-1}
\end{array}\right\} .
$$

These cosets contribute zero. 
$\mathrm{VIII}^{\prime} . \quad\left(\begin{array}{cccc}p^{2} & a & e p+a b & d \\ & p & b p & e \\ & & p^{2} & -a \\ & & & p\end{array}\right),\left\{a, b, d, e \bmod p, p \nmid a, \kappa=\left(\frac{a}{p}\right)\right\}$.

These cosets contribute $\mathbb{N} p^{7 / 2} a^{p^{-1}[\rho]}\left(n_{1} p, n_{2} p^{-1}\right) g\left(n_{2} p^{-1}, p\right)$.

$$
\operatorname{IX}^{\prime} .\left(\begin{array}{cccc}
p^{2} & & e p & d \\
& p & b & e \\
& p^{2} & \\
& & p
\end{array}\right),\left\{d, e \bmod p, b \bmod p^{2}, p \nmid b d, \kappa=\left(\frac{b d^{2}}{p}\right)\right\} .
$$

These cosets contribute zero.

$$
\mathrm{X}^{\prime} .\left(\begin{array}{cccc}
p^{2} & & e p^{2} & d \\
& 1 & b & e \\
& & p^{3} & \\
& & p
\end{array}\right),\left\{d, e \bmod p, b \bmod p^{3}, p \nmid d, \kappa=\left(\frac{d}{p}\right)^{-1}\right\} .
$$

These cosets contribute zero.

$$
\mathrm{XI}^{\prime} .\left(\begin{array}{cccc}
p & a p & & d \\
& p^{3} & & \\
& & 1 & -a \\
& & & p^{2}
\end{array}\right),\left\{a, d \bmod p^{2}, p \nmid d, \kappa=\left(\frac{d}{p}\right)\right\} .
$$

These cosets contribute zero.

$$
\mathrm{XII}^{\prime} . \quad\left(\begin{array}{cccc}
p & a & c & d \\
& p^{2} & & c p \\
& & p & -a \\
& & & p^{2}
\end{array}\right),\left\{c \bmod p, a, d \bmod p^{2}, p \nmid a, \kappa=\left(\frac{a}{p}\right)^{-1}\right\} .
$$

These cosets contribute $\mathbb{N} p^{7 / 2} a^{p[\rho]}\left(n_{1} p^{-1}, n_{2} p\right) \overline{g\left(n_{2}, p\right)}$.

$$
\mathrm{XIII}^{\prime} .\left(\begin{array}{cccc}
p & a p & c & d \\
& p^{2} & b & c p-a b \\
& & p & -a p \\
& & & p^{2}
\end{array}\right),\left\{\begin{array}{l}
a, b, c \bmod p, d \bmod p^{2}, p \nmid b(d+a c), \\
\kappa=\left(\frac{b^{2}(d+a c)}{p}\right)
\end{array}\right\} .
$$

These cosets contribute zero.

$$
\mathrm{XIV}^{\prime} .\left(\begin{array}{cccc}
p & a & c & d \\
& p & b p & c-a b \\
& & p^{2} & -a p \\
& & & p^{2}
\end{array}\right),\left\{\begin{array}{l}
a, b \bmod p, c, d \bmod p^{2} \\
p \nmid a(c-a b), \kappa=\left(\frac{c-a b}{p}\right)^{-1}
\end{array}\right\} .
$$

These cosets contribute zero.

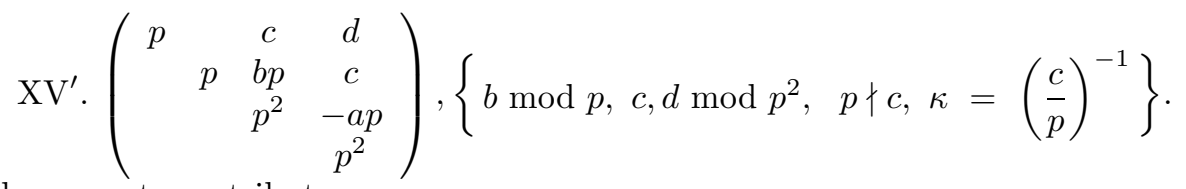

These cosets contribute zero.

$$
\mathrm{XVI}^{\prime} .\left(\begin{array}{cccc}
p & & c & d \\
& p & b & c \\
& & p^{2} & \\
& & p^{2}
\end{array}\right),\left\{b, c, d \bmod p^{2}, \quad p \nmid b\left(c^{2}-b d\right), \kappa=\left(\frac{c^{2}-b d}{p}\right)\right\} .
$$

These cosets contribute zero. 


$$
\text { XVII'. }\left(\begin{array}{cccc}
p & & e p & d \\
& 1 & b & e \\
& & p^{3} & \\
& & p^{2}
\end{array}\right),\left\{d, e \bmod p^{2}, b \bmod p^{3}, p \nmid d, \kappa=\left(\frac{d}{p}\right)\right\} .
$$

These cosets contribute zero.

$$
\text { XVIII'. }\left(\begin{array}{cccc}
1 & a & & d \\
& p^{3} & & \\
& & 1 & -a \\
& & & p^{3}
\end{array}\right),\left\{a, d \bmod p^{3}, \kappa=1\right\} .
$$

These cosets contribute $\mathbb{N} p^{9 / 2} a^{p^{3}[\rho]}\left(n_{1} p^{-3}, n_{2} p^{3}\right)$.

$$
\mathrm{XIX}^{\prime} .\left(\begin{array}{cccc}
1 & a & c & d \\
& p^{2} & b & c p^{2}-a b \\
& & p & -a p \\
& & & p^{3}
\end{array}\right),\left\{\begin{array}{l}
b, c \bmod p, a \bmod p^{2}, d \bmod p^{3}, \\
p \nmid b, \kappa=\left(\frac{b}{p}\right)^{-1}
\end{array}\right\} \text {. }
$$

These cosets contribute $\mathbb{N} p^{7 / 2} a^{p[\rho]}\left(n_{1} p^{-1}, n_{2} p^{2}\right) \overline{g\left(n_{1} p^{-1}, p\right)}$.

$$
\mathrm{XX}^{\prime} .\left(\begin{array}{cccc}
1 & a & c & d \\
& p & b & c p-a b \\
& & p^{2} & -a p^{2} \\
& & & p^{3}
\end{array}\right),\left\{\begin{array}{l}
a \bmod p, b, c \bmod p^{2}, d \bmod p^{3}, \\
p \nmid b, \kappa=\left(\frac{b}{p}\right)
\end{array}\right\} .
$$

These cosets contribute $\mathbb{N} p^{7 / 2} a^{p^{-1}[\rho]}\left(n_{1} p, n_{2} p\right) g\left(n_{1}, p\right)$.

$$
\mathrm{XXI}^{\prime} .\left(\begin{array}{cccc}
1 & & c & d \\
& 1 & b & c \\
& & p^{3} & \\
& & & p^{3}
\end{array}\right),\left\{b, c, d \bmod p^{3}, \kappa=1\right\} .
$$

These cosets contribute $\mathbb{N} p^{9 / 2} a^{p^{-3}[\rho]}\left(n_{1} p^{3}, n_{2}\right)$.

\section{The Euler PRoduct}

The expression of the Shimura type Rankin-Selberg integral (3.1) as a double Dirichlet series $D(s)$ times $G(s)$ in (3.2) is valid for any cubic metaplectic automorphic form on GL(3) that satisfies the hypothesis of Lemma 2.2. However, by taking $f=\Theta$, the cubic theta function, it will be shown that $D(s)$ has an expression as an Euler product. Note that $\Theta$ satisfies the hypothesis of Lemma 2.2, since $\Theta\left(\gamma \tau_{3}\right)=\Theta\left(\tau_{3}\right)$ for all $\gamma \in \mathrm{SL}(3, \mathcal{O})$ (see $(2.17)$ of $[\mathrm{BH} 1]$ ). The choice of $\Theta$ here (and the similar use of theta functions by Bump-Hoffstein [BH2] and FriedbergWong $[\mathrm{FrW}]$ ) follows Shimura's original technique [Shi] in the sense that the theta function picks off the Whittaker-Fourier coefficients of $\phi$ that allow $D(s)$ to have an Euler product. The goal, in this section, is to prove

Theorem 5.1. Suppose that $\phi$ is a cubic generic cusp form on $\operatorname{GSp}(4)$ that is an eigenfunction of the Hecke operators $T_{p^{6}}, T_{p^{3}}$ for each prime $p \in \mathcal{O}$ with $(p, \lambda)=1$, with eigenvalues given by 


$$
T_{p^{6}} \cdot \phi=\left(\mathbb{N} p^{6} \mu_{p^{6}}-\mathbb{N} p^{6}-\mathbb{N} p^{4}\right)
$$

and

$$
T_{p^{3}} \cdot \phi=\mathbb{N} p^{9 / 2} \mu_{p^{3}} \phi .
$$

Let $D(s)$ be the Dirichlet series constructed as in (3.3) using the cubic theta function $\Theta$. Then for $\operatorname{Re}(s)>3 / 4$,

$$
\zeta_{K}(12 s-4) D(s)=R\left(6 s-\frac{5}{2}\right) R_{\lambda}\left(6 s-\frac{5}{2}\right),
$$

where

$$
R(s)=\prod_{(p, \lambda)=1}\left(1-\mu_{p^{3}} \mathbb{N} p^{-s}+\mu_{p^{6}} \mathbb{N} p^{-2 s}-\mu_{p^{3}} \mathbb{N} p^{-3 s}+\mathbb{N} p^{-4 s}\right)^{-1}
$$

and

$$
R_{\lambda}(s)=\left(1-\mathbb{N} \lambda^{-2 s-1}\right)^{-1} \sum_{\rho \in \Gamma_{2}(3) \backslash \Gamma_{2} / \Gamma_{2, \infty}} \sum_{\begin{array}{c}
n_{1} \in(\lambda)^{-5} \\
n_{2} \in(\lambda)^{-3} \\
p \mid 27 n_{1} n_{2} \Rightarrow(p, \lambda)>1
\end{array}} \frac{a_{n_{1}, n_{2}}^{\rho} \overline{\theta_{n_{2}, n_{1}}^{\rho}}}{\mathbb{N}\left(n_{1} n_{2}^{2}\right)^{(s+3) / 3}} .
$$

Proof. First fix a prime $p \equiv 1 \bmod (3)$, and take $n_{1} \in(\lambda)^{-5}, n_{2} \in(\lambda)^{-3}$ such that $p \nmid 27 n_{1} n_{2}$. Let $x=\mathbb{N} p, z=\mathbb{N} p^{-s}$, and let $\mu_{1}, \mu_{2}$ be the eigenvalues of $\phi$ corresponding to $T_{p^{6}}$, and $T_{p^{3}}$ respectively. Also put

$$
F\left(z^{6}\right)=1-\frac{\mu_{2}}{x^{2}} z^{6}+\left(\frac{\mu_{1}}{x}+x^{3}+x^{5}\right) z^{12}-\mu_{2} x^{3} z^{18}+x^{10} z^{24} .
$$

Then Theorem 5.1 will follow from

\section{Lemma 5.2.}

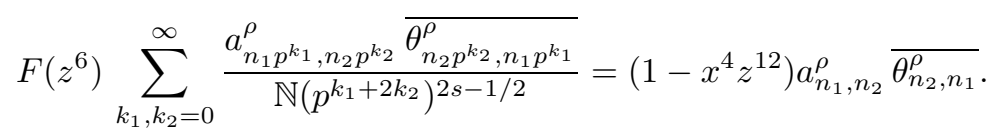

Proof. Let $G(6 n)$ be the coefficient of $z^{6 n}$ in the left-hand side of (5.1). Proposition 2.3 implies the equality

$$
\begin{aligned}
\sum_{k_{1}, k_{2}=0}^{\infty} & \frac{a_{n_{1} p^{k_{1}}, n_{2} p^{k_{2}}}^{\rho} \overline{\theta_{n_{2} p^{k_{2}}, n_{1} p^{k_{1}}}^{\rho}}}{\mathbb{N}\left(p^{k_{1}+2 k_{2}}\right)^{2 s-1 / 2}} \\
= & \sum_{k_{1}, k_{2}=0}^{\infty} \overline{\theta_{n_{2}, n_{1}}} x^{\frac{5}{2} k_{1}+4 k_{2}} z^{6 k_{1}+12 k_{2}} \\
& \times\left(a_{n_{1} p^{3 k_{1}}, n_{2} p^{3 k_{2}}}^{\rho}+g\left(n_{2}, p\right) x^{3 / 2} z^{6} a_{n_{1} p^{3 k_{1}+1}, n_{2} p^{3 k_{2}+1}}^{\rho}\right) .
\end{aligned}
$$


Now define

$$
B\left(k_{1}, k_{2}\right)=\overline{\theta_{n_{2}, n_{1}}^{\rho}} x^{\frac{5}{2}} k_{1}+4 k_{2} a_{n_{1} p^{3 k_{1}}, n_{2} p^{3 k_{2}}}^{\rho}
$$

and

$$
C\left(k_{1}, k_{2}\right)=\overline{\theta_{n_{2}, n_{1}}^{\rho}} x^{\frac{5}{2} k_{1}+4 k_{2}+\frac{3}{2}} g\left(n_{2}, p\right) a_{n_{1} p^{3 k_{1}+1}, n_{2} p^{3 k_{2}+1}}^{\rho} .
$$

Then the coefficient of $z^{6 n}$ appearing in the right-hand side of (5.2), hereafter designated $G^{\prime}(6 n)$, is given by

$$
\begin{aligned}
G^{\prime}(0)= & B(0,0), \\
G^{\prime}(6)= & B(1,0)+C(0,0), \\
G^{\prime}(6 n)= & B(n, 0)+C(n-1,0)+B(n-2,1)+C(n-3,1) \\
& +\ldots+ \begin{cases}B\left(0, \frac{n}{2}\right)+C\left(1, \frac{n-2}{2}\right) & \text { if } n \geq 2 \text { is even, } \\
B\left(1, \frac{n-1}{2}\right)+C\left(0, \frac{n-1}{2}\right) & \text { if } n \geq 2 \text { is odd. }\end{cases}
\end{aligned}
$$

Consequently, by defining $G^{\prime}(6 n)=0$ if $n<0$, for all $n$ we get

$$
\begin{aligned}
G(6 n)= & G^{\prime}(6 n)-\frac{\mu_{2}}{x^{2}} G^{\prime}(6 n-6)+\left(\frac{\mu_{1}}{x}+x^{3}+x^{5}\right) G^{\prime}(6 n-12) \\
& -\mu_{2} x^{3} G^{\prime}(6 n-18)+x^{10} G^{\prime}(6 n-24) .
\end{aligned}
$$

Now it can be checked explicitly for $n=0,1,2,3,4,5$ that $G(6 n)$ has the form suggested in (5.1). By virtue of (5.3) and (5.4) it suffices to know $\mu_{1} G^{\prime}(6 n)$ and $\mu_{2} G^{\prime}(6 n)$ from Theorem 4.2, for each $n$ under consideration. Using Theorem 4.2 and Proposition 2.3 one finds the following:

$$
\begin{aligned}
G^{\prime}(0)= & \overline{\theta_{n_{2}, n_{1}}^{\rho}} a_{n_{1}, n_{2}}^{\rho}, \\
\mu_{1} G^{\prime}(0)= & \overline{\theta_{n_{2}, n_{1}}^{\rho}}\left[-x^{4} a_{n_{1}, n_{2}}^{\rho}+x^{6} a_{n_{1}, n_{2} p^{3}}^{\rho}\right. \\
& \left.+x^{5} a_{n_{1} p^{2}, n_{2} p}^{\rho} \overline{g\left(n_{1}, p\right)}+x^{4} a_{n_{1} p^{2}, n_{2}}^{\rho} g\left(n_{1}, p\right)^{2}\right], \\
\mu_{2} G^{\prime}(0)= & \overline{\theta_{n_{2}, n_{1}}^{\rho}}\left[x^{9 / 2} a_{n_{1} p^{3}, n_{2}}^{\rho}+x^{7 / 2} a_{n_{1} p, n_{2} p}^{\rho} g\left(n_{1}, p\right)\right], \\
G^{\prime}(6)= & \overline{\theta_{n_{2}, n_{1}}^{\rho}}\left[x^{5 / 2} a_{n_{1} p^{3}, n_{2}}^{\rho}+x^{3 / 2} a_{n_{1} p, n_{2} p}^{\rho} g\left(n_{1}, p\right)\right], \\
\mu_{1} G^{\prime}(6)= & \overline{\theta_{n_{2}, n_{1}}^{\rho}}\left[\left(x^{2}+x-1\right) x^{13 / 2} a_{n_{1} p^{3}, n_{2}}^{\rho}+x^{17 / 2} a_{n_{1} p^{3}, n_{2} p^{3}}^{\rho}\right. \\
& +x^{15 / 2} a_{n_{1} p^{5}, n_{2} p}^{\rho} \overline{g\left(n_{1}, p\right)}+\left(x^{2}+x-1\right) x^{11 / 2} a_{n_{1} p, n_{2} p}^{\rho} g\left(n_{1}, p\right) \\
& \left.+x^{15 / 2} a_{n_{1} p, n_{2} p^{4}}^{\rho} g\left(n_{1}, p\right)+x^{13 / 2} a_{n_{1} p^{5}, n_{2}}^{\rho} g\left(n_{1}, p\right)^{2}\right],
\end{aligned}
$$




$$
\begin{aligned}
& \mu_{2} G^{\prime}(6)=\overline{\theta_{n_{2}, n_{1}}^{\rho}}\left[(x+1) x^{6} a_{n_{1}, n_{2}}^{\rho}+(x+1) x^{6} a_{n_{1}, n_{2} p^{3}}^{\rho}+x^{7} a_{n_{1} p^{6}, n_{2}}^{\rho}\right. \\
& \left.+x^{6} a_{n_{1} p^{2}, n_{2} p}^{\rho} \overline{g\left(n_{1}, p\right)}+x^{6} a_{n_{1} p^{4}, n_{2} p}^{\rho} g\left(n_{1}, p\right)+x^{5} a_{n_{1} p^{2}, n_{2}}^{\rho} g\left(n_{1}, p\right)^{2}\right], \\
& G^{\prime}(12)=\overline{\theta_{n_{2}, n_{1}}}\left[x^{4} a_{n_{1}, n_{2} p^{3}}^{\rho}+x^{5} a_{n_{1} p^{6}, n_{2}}^{\rho}+x^{4} a_{n_{1} p^{4}, n_{2} p}^{\rho} g\left(n_{1}, p\right)\right], \\
& \mu_{1} G^{\prime}(12)=\overline{\theta_{n_{2}, n_{1}}^{\rho}}\left[x^{10} a_{n_{1}, n_{2}}^{\rho}+\left(x^{3}+x^{2}+x-1\right) x^{8} a_{n_{1}, n_{2} p^{3}}^{\rho}+x^{10} a_{n_{1}, n_{2} p^{6}}^{\rho}\right. \\
& +\left(x^{2}+x-1\right) x^{9} a_{n_{1} p^{6}, n_{2}}^{\rho}+x^{11} a_{n_{1} p^{6}, n_{2} p^{3}}^{\rho}+x^{10} a_{n_{1} p^{2}, n_{2} p}^{\rho} \overline{g\left(n_{1}, p\right)} \\
& +x^{10} a_{n_{1} p^{8}, n_{2} p}^{\rho} \overline{g\left(n_{1}, p\right)}+\left(x^{2}+x-1\right) x^{8} a_{n_{1} p^{4}, n_{2} p}^{\rho} g\left(n_{1}, p\right) \\
& +x^{10} a_{n_{1} p^{4}, n_{2} p^{4}}^{\rho} g\left(n_{1}, p\right)+x^{9} a_{n_{1} p^{2}, n_{2}}^{\rho} g\left(n_{1}, p\right)^{2} \\
& \left.+x^{9} a_{n_{1} p^{8}, n_{2}}^{\rho} g\left(n_{1}, p\right)^{2}\right], \\
& \mu_{2} G^{\prime}(12)=\overline{\theta_{n_{2}, n_{1}}^{\rho}}\left[(x+1) x^{17 / 2} a_{n_{1} p^{3}, n_{2}}^{\rho}+(x+1) x^{17 / 2} a_{n_{1} p^{3}, n_{2} p^{3}}^{\rho}\right. \\
& +x^{19 / 2} a_{n_{1} p^{9}, n_{2}}^{\rho}+x^{17 / 2} a_{n_{1} p^{5}, n_{2} p}^{\rho} \overline{g\left(n_{1}, p\right)} \\
& +(x+1) x^{15 / 2} a_{n_{1} p, n_{2} p}^{\rho} g\left(n_{1}, p\right)+(x+1) x^{15 / 2} a_{n_{1} p, n_{2} p^{4}}^{\rho} g\left(n_{1}, p\right) \\
& \left.+x^{17 / 2} a_{n_{1} p^{7}, n_{2} p}^{\rho} g\left(n_{1}, p\right)+x^{15 / 2} a_{n_{1} p^{5}, n_{2}}^{\rho} g\left(n_{1}, p\right)^{2}\right], \\
& G^{\prime}(18)=\overline{\theta_{n_{2}, n_{1}}^{\rho}}\left[x^{13 / 2} a_{n_{1} p^{3}, n_{2} p^{3}}^{\rho}+x^{15 / 2} a_{n_{1} p^{9}, n_{2}}^{\rho}\right. \\
& \left.+x^{11 / 2} a_{n_{1} p, n_{2} p^{4}}^{\rho} g\left(n_{1}, p\right)+x^{13 / 2} a_{n_{1} p^{7}, n_{2} p}^{\rho} g\left(n_{1}, p\right)\right], \\
& \mu_{1} G^{\prime}(18)=\overline{\theta_{n_{2}, n_{1}}^{\rho}}\left[x^{25 / 2} a_{n_{1} p^{3}, n_{2}}^{\rho}+\left(x^{3}+x^{2}+x-1\right) x^{21 / 2} a_{n_{1} p^{3}, n_{2} p^{3}}^{\rho}\right. \\
& +x^{25 / 2} a_{n_{1} p^{3}, n_{2} p^{6}}^{\rho}+\left(x^{2}+x-1\right) x^{23 / 2} a_{n_{1} p^{9}, n_{2}}^{\rho}+x^{27 / 2} a_{n_{1} p^{9}, n_{2} p^{3}}^{\rho} \\
& +x^{25 / 2} a_{n_{1} p^{5}, n_{2} p}^{\rho} \overline{g\left(n_{1}, p\right)}+x^{25 / 2} a_{n_{1} p^{11}, n_{2} p}^{\rho} \overline{g\left(n_{1}, p\right)} \\
& +x^{23 / 2} a_{n_{1} p, n_{2} p}^{\rho} g\left(n_{1}, p\right)+\left(x^{3}+x^{2}+x-1\right) x^{19 / 2} a_{n_{1} p, n_{2} p^{4}}^{\rho} g\left(n_{1}, p\right) \\
& +x^{23 / 2} a_{n_{1} p, n_{2} p^{7}}^{\rho}+\left(x^{2}+x-1\right) x^{21 / 2} a_{n_{1} p^{7}, n_{2} p}^{\rho} g\left(n_{1}, p\right) \\
& +x^{25 / 2} a_{n_{1} p^{7}, n_{2} p^{4}}^{\rho}+x^{23 / 2} a_{n_{1} p^{5}, n_{2}}^{\rho} g\left(n_{1}, p\right)^{2} \\
& \left.+x^{23 / 2} a_{n_{1} p^{11}, n_{2}}^{\rho} g\left(n_{1}, p\right)^{2}\right], \\
& \mu_{2} G^{\prime}(18)=\overline{\theta_{n_{2}, n_{1}}^{\rho}}\left[(x+1) x^{10} a_{n_{1}, n_{2} p^{3}}^{\rho}+(x+1) x^{10} a_{n_{1}, n_{2} p^{6}}^{\rho}\right. \\
& +(x+1) x^{11} a_{n_{1} p^{6}, n_{2}}^{\rho}+(x+1) x^{11} a_{n_{1} p^{6}, n_{2} p^{3}}^{\rho}+x^{12} a_{n_{1} p^{12}, n_{2}}^{\rho} \\
& +x^{11} a_{n_{1} p^{8}, n_{2} p}^{\rho} \overline{g\left(n_{1}, p\right)}+(x+1) x^{10} a_{n_{1} p^{4}, n_{2} p}^{\rho} g\left(n_{1}, p\right) \\
& +(x+1) x^{10} a_{n_{1} p^{4}, n_{2} p^{4}}^{\rho} g\left(n_{1}, p\right)+x^{11} a_{n_{1} p^{10}, n_{2} p}^{\rho} g\left(n_{1}, p\right) \\
& \left.+x^{10} a_{n_{1} p^{8}, n_{2}}^{\rho} g\left(n_{1}, p\right)^{2}\right] \text {, } \\
& G^{\prime}(24)=\overline{\theta_{n_{2}, n_{1}}^{\rho}}\left[x^{8} a_{n_{1}, n_{2} p^{6}}^{\rho}+x^{9} a_{n_{1} p^{6}, n_{2} p^{3}}^{\rho}+x^{10} a_{n_{1} p^{12}, n_{2}}^{\rho}\right. \\
& \left.+x^{8} a_{n_{1} p^{4}, n_{2} p^{4}}^{\rho} g\left(n_{1}, p\right)+x^{9} a_{n_{1} p^{10}, n_{2} p}^{\rho} g\left(n_{1}, p\right)\right] \text {, }
\end{aligned}
$$




$$
\begin{aligned}
& \mu_{1} G^{\prime}(24)=\overline{\theta_{n_{2}, n_{1}}^{\rho}}\left[x^{14} a_{n_{1}, n_{2} p^{3}}^{\rho}+\left(x^{3}+x^{2}+x-1\right) x^{12} a_{n_{1}, n_{2} p^{6}}^{\rho}+x^{14} a_{n_{1}, n_{2} p^{9}}^{\rho}\right. \\
& +x^{15} a_{n_{1} p^{6}, n_{2}}^{\rho}+\left(x^{3}+x^{2}+x-1\right) x^{13} a_{n_{1} p^{6}, n_{2} p^{3}}^{\rho}+x^{15} a_{n_{1} p^{6}, n_{2} p^{6}}^{\rho} \\
& +\left(x^{2}+x-1\right) x^{14} a_{n_{1} p^{12}, n_{2}}^{\rho}+x^{16} a_{n_{1} p^{12}, n_{2} p^{3}}^{\rho}+x^{15} a_{n_{1} p^{8}, n_{2} p}^{\rho} \overline{g\left(n_{1}, p\right)} \\
& +x^{15} a_{n_{1} p^{14}, n_{2} p}^{\rho g\left(n_{1}, p\right)}+x^{14} a_{n_{1} p^{4}, n_{2} p}^{\rho} g\left(n_{1}, p\right) \\
& +\left(x^{3}+x^{2}+x-1\right) x^{12} a_{n_{1} p^{4}, n_{2} p^{4}}^{\rho} g\left(n_{1}, p\right)+x^{14} a_{n_{1} p^{4}, n_{2} p^{7}}^{\rho} g\left(n_{1}, p\right) \\
& +\left(x^{2}+x-1\right) x^{13} a_{n_{1} p^{10}, n_{2} p}^{\rho} g\left(n_{1}, p\right)+x^{15} a_{n_{1} p^{10}, n_{2} p^{4}}^{\rho} g\left(n_{1}, p\right) \\
& \left.+x^{14} a_{n_{1} p^{8}, n_{2}}^{\rho} g\left(n_{1}, p\right)^{2}+x^{14} a_{n_{1} p^{14}, n_{2}}^{\rho} g\left(n_{1}, p\right)^{2}\right], \\
& \mu_{2} G^{\prime}(24)=\overline{\theta_{n_{2}, n_{1}}^{\rho}}\left[(x+1) x^{25 / 2} a_{n_{1} p^{3}, n_{2} p^{3}}^{\rho}+(x+1) x^{25 / 2} a_{n_{1} p^{3}, n_{2} p^{6}}^{\rho}\right. \\
& +(x+1) x^{27 / 2} a_{n_{1} p^{9}, n_{2}}^{\rho}+(x+1) x^{27 / 2} a_{n_{1} p^{9}, n_{2} p^{3}}^{\rho}+x^{29 / 2} a_{n_{1} p^{15}, n_{2}}^{\rho} \\
& +x^{27 / 2} a_{n_{1} p^{11}, n_{2} p}^{\rho} \overline{g\left(n_{1}, p\right)}+(x+1) x^{23 / 2} a_{n_{1} p, n_{2} p^{4}}^{\rho} g\left(n_{1}, p\right) \\
& +(x+1) x^{23 / 2} a_{n_{1} p, n_{2} p^{7}}^{\rho} g\left(n_{1}, p\right)+(x+1) x^{25 / 2} a_{n_{1} p^{7}, n_{2} p}^{\rho} g\left(n_{1}, p\right) \\
& +(x+1) x^{25 / 2} a_{n_{1} p^{7}, n_{2} p^{4}}^{\rho} g\left(n_{1}, p\right)+x^{27 / 2} a_{n_{1} p^{13}, n_{2} p}^{\rho} g\left(n_{1}, p\right) \\
& \left.+x^{25 / 2} a_{n_{1} p^{11}, n_{2}}^{\rho} g\left(n_{1}, p\right)^{2}\right], \\
& G^{\prime}(30)=\overline{\theta_{n_{2}, n_{1}}^{\rho}}\left[x^{21 / 2} a_{n_{1} p^{3}, n_{2} p^{6}}^{\rho}+x^{23 / 2} a_{n_{1} p^{9}, n_{2} p^{3}}^{\rho}\right. \\
& +x^{25 / 2} a_{n_{1} p^{15}, n_{2}}^{\rho}+x^{19 / 2} a_{n_{1} p, n_{2} p^{7}}^{\rho} g\left(n_{1}, p\right) \\
& \left.+x^{21 / 2} a_{n_{1} p^{7}, n_{2} p^{4}}^{\rho} g\left(n_{1}, p\right)+x^{23 / 2} a_{n_{1} p^{13}, n_{2} p}^{\rho} g\left(n_{1}, p\right)\right] .
\end{aligned}
$$

So by using these formulae in (5.4) it follows that

$$
\begin{gathered}
G(0)=a_{n_{1}, n_{2}}^{\rho} \overline{\theta_{n_{2}, n_{1}}^{\rho}}, \\
G(12)=-x^{4} \overline{\theta_{n_{2}, n_{1}}^{\rho}} a_{n_{1}, n_{2}}^{\rho},
\end{gathered}
$$

and

$$
G(6)=G(18)=G(24)=G(30)=0,
$$

as required in (5.1).

To finish the proof of Lemma 5.2 it remains to show that $G(6 n)=0$ for $n \geq 6$. First, write

$$
G(6 n)=\sum_{i=1}^{6} G_{i}(6 n)
$$

where

$G_{1}(6 n)$ is the sum of coefficients of $z^{6 n}$

such that $k_{1} \equiv k_{2} \equiv 0 \bmod (3), k_{1}, k_{2} \geq 3$,

$G_{2}(6 n)$ is the sum of coefficients of $z^{6 n}$

such that $k_{1} \equiv k_{2} \equiv 1 \bmod (3), k_{1}, k_{2} \geq 3$,

$G_{3}(6 n)$ is the sum of coefficients of $z^{6 n}$ such that $k_{1}=0$,

$G_{4}(6 n)$ is the sum of coefficients of $z^{6 n}$ such that $k_{1}=1$,

$G_{5}(6 n)$ is the sum of coefficients of $z^{6 n}$ such that $k_{2}=0, k_{1} \neq 0$ or 1 ,

$G_{6}(6 n)$ is the sum of coefficients of $z^{6 n}$ such that $k_{2}=1, k_{1} \neq 0$ or 1 . 
Then it suffices to prove that, for all $n \geq 6$,

$$
\sum_{i=1}^{4} G_{i}(6 n)=0
$$

and

$$
G_{5}(6 n)=G_{6}(6 n)=0 .
$$

Equation (5.6) will be proved first. To this end, write

$$
G_{5}(6 n)=\sum_{j=0}^{4} \sum_{\substack{k_{1}=3 n-3 j \\ k_{1} \geq 2}} \overline{\theta^{\rho}\left(n_{2}, n_{1} p^{k_{1}}\right)} A_{j}\left(k_{1}, 0\right) x^{k_{1} / 2},
$$

where

$$
\begin{aligned}
& A_{0}\left(k_{1}, k_{2}\right)=a^{\rho}\left(n_{1} p^{k_{1}}, n_{2} p^{k_{2}}\right), \\
& A_{1}\left(k_{1}, k_{2}\right)=\frac{-\mu_{2}}{x^{2}} A_{0}\left(k_{1}, k_{2}\right), \\
& A_{2}\left(k_{1}, k_{2}\right)=\left(\frac{\mu_{1}}{x}+x^{3}+x^{5}\right) A_{0}\left(k_{1}, k_{2}\right), \\
& A_{3}\left(k_{1}, k_{2}\right)=-\mu_{2} x^{3} A_{0}\left(k_{1}, k_{2}\right), \\
& A_{4}\left(k_{1}, k_{2}\right)=x^{10} A_{0}\left(k_{1}, k_{2}\right) .
\end{aligned}
$$

Then using Proposition 2.3, (5.7) becomes

$$
\sum_{j=0}^{4} \overline{\theta_{n_{2}, n_{1}}^{\rho}} x^{5(n-j) / 2} A_{j}(3(n-j), 0) .
$$

So to prove $G_{5}(6 n)=0$ for all $n \geq 6$, it suffices to prove

$$
\sum_{j=0}^{4} x^{5(n-j) / 2} A_{j}(3(n-j), 0)=0 .
$$

Now, using the definition of the $A_{j}$ and Theorem 4.2, one can find that

$$
\begin{aligned}
A_{1}(3 n-3,0) & =-x^{5 / 2}\left[a^{\rho}\left(n_{1} p^{3 n-6}, n_{2}\right)+a^{\rho}\left(n_{1} p^{3 n-6}, n_{2} p^{3}\right)+a^{\rho}\left(n_{1} p^{3 n}, n_{2}\right)\right] \\
& -x^{3 / 2} \frac{\bar{g}\left(n_{2}, p\right)}{a^{\rho}}\left(n_{1} p^{3 n-4}, n_{2} p\right),
\end{aligned}
$$

$$
\begin{aligned}
A_{2}(3 n-6,0) & =\left[x^{3}+x^{5}\right] a^{\rho}\left(n_{1} p^{3 n-6}, n_{2}\right)+x^{5} a^{\rho}\left(n_{1} p^{3 n-12}, n_{2} p^{3}\right) \\
& +x^{5} a^{\rho}\left(n_{1} p^{3 n-6}, n_{2} p^{3}\right)+x^{4} \overline{g\left(n_{2}, p\right)} a^{\rho}\left(n_{1} p^{3 n-10}, n_{2} p\right), \\
& +x^{4} \overline{g\left(n_{2}, p\right)} a^{\rho}\left(n_{1} p^{3 n-4}, n_{2} p\right)+x^{3}\left(x^{2}-1\right) a^{\rho}\left(n_{1} p^{3 n-6}, n_{2}\right),
\end{aligned}
$$

$$
\begin{aligned}
A_{3}(3 n & -9,0) \\
= & -x^{15 / 2}\left[a^{\rho}\left(n_{1} p^{3 n-12}, n_{2}\right)+a^{\rho}\left(n_{1} p^{3 n-12}, n_{2} p^{3}\right)+a^{\rho}\left(n_{1} p^{3 n-6}, n_{2}\right)\right] \\
& -x^{13 / 2} \frac{2\left(n_{2}, p\right)}{a^{\rho}}\left(n_{1} p^{3 n-10}, n_{2} p\right) .
\end{aligned}
$$


Then using (5.9)-(5.11), (5.8) follows immediately. Next, by writing

$$
G_{6}(6 n)=\sum_{j=0}^{4} \overline{\theta_{n_{2}, n_{1}}^{\rho}} g\left(n_{2}, p\right) x^{5(n-j) / 2} A_{j}(3(n-j), 1),
$$

as in (5.8), it is straightforward to verify that (5.6) holds in this case as well. To prove (5.5), first write

$$
G_{i}(6 n)=\sum_{j=0}^{4} \sum_{\left(k_{1}, k_{2}\right) \in K_{j}^{i, n}} \overline{\theta^{\rho}\left(n_{2} p^{k_{2}}, n_{1} p^{k_{1}}\right)} A_{j}\left(k_{1}, k_{2}\right) x^{\left(k_{1}+2 k_{2}\right) / 2},
$$

where

$$
\begin{aligned}
K_{j}^{1, n} & =\left\{\left(k_{1}, k_{2}\right) \in \mathbb{Z}^{2} \mid k_{1} \equiv k_{2} \equiv 0 \bmod (3), k_{1}, k_{2} \geq 3, k_{1}+2 k_{2}=3(n-j)\right\}, \\
K_{j}^{2, n} & =\left\{\left(k_{1}, k_{2}\right) \in \mathbb{Z}^{2} \mid k_{1} \equiv k_{2} \equiv 1 \bmod (3), k_{1}, k_{2} \geq 3, k_{1}+2 k_{2}=3(n-j)\right\}, \\
K_{j}^{3, n} & =\left\{\left(0, k_{2}\right) \in \mathbb{Z}^{2} \mid k_{2} \equiv 0 \bmod (3), 2 k_{2}=3(n-j)\right\}, \\
K_{j}^{4, n} & =\left\{\left(1, k_{2}\right) \in \mathbb{Z}^{2} \mid k_{2} \equiv 1 \bmod (3), 1+2 k_{2}=3(n-j)\right\} .
\end{aligned}
$$

The congruence conditions on $k_{2}$ for $K_{j}^{3, n}$ and $K_{j}^{4, n}$ are included to ensure that the coefficient of $\theta^{\rho}\left(n_{2} p^{k_{2}}, n_{1} p^{k_{1}}\right)$ is not zero. Then, consider the

Claim. For $i \in\{1,2,3,4\}$ and $n \geq 6$, the coefficient of $a^{\rho}\left(n_{1} p^{k_{1}}, n_{2} p^{k_{2}+3}\right)$ in $G_{i}(6(n+2))$ is equal to $x^{4}$ times the coefficient of $a^{\rho}\left(n_{1} p^{k_{1}}, n_{2} p^{k_{2}}\right)$ in $G_{i}(6 n)$.

By induction, this claim reduces (5.5) to the cases of $n=6$ and $n=7$. To prove the claim, notice that (2.3) implies

$$
\theta^{\rho}\left(n_{2} p^{k_{2}}, n_{1} p^{k_{1}}\right)= \begin{cases}x^{\left(k_{1}+k_{2}\right) / 3} \theta_{n_{2}, n_{1}}^{\rho} & \text { if }\left(k_{1}, k_{2}\right) \in K_{j}^{1, n}, \\ x^{\left(k_{1}+k_{2}-2\right) / 3} \overline{g\left(n_{2}, p\right)} \theta_{n_{2}, n_{1}}^{\rho} & \text { if }\left(k_{1}, k_{2}\right) \in K_{j}^{2, n}, \\ x^{k_{2} / 3} \theta_{n_{2}, n_{1}}^{\rho} & \text { if }\left(k_{1}, k_{2}\right) \in K_{j}^{3, n}, \\ x^{k_{2} / 3} \overline{g\left(n_{2}, p\right)} \theta_{n_{2}, n_{1}}^{\rho} & \text { if }\left(k_{1}, k_{2}\right) \in K_{j}^{4, n} .\end{cases}
$$

Therefore,

$$
G_{1}(6 n)=\sum_{j=0}^{4} \sum_{\left(k_{1}, k_{2}\right) \in K_{j}^{1, n}} \overline{\theta_{n_{2}, n_{1}}^{\rho}} A_{j}\left(k_{1}, k_{2}\right) x^{\left(k_{1}+k_{2}\right) / 3} x^{\left(k_{1}+2 k_{2}\right) / 2}
$$

$$
\begin{aligned}
& G_{2}(6 n)=\sum_{j=0}^{4} \sum_{\left(k_{1}, k_{2}\right) \in K_{j}^{2, n}} \overline{\theta_{n_{2}, n_{1}}^{\rho}} g\left(n_{2}, p\right) A_{j}\left(k_{1}, k_{2}\right) x^{\left(k_{1}+k_{2}-2\right) / 3} x^{\left(k_{1}+2 k_{2}\right) / 2}, \\
& G_{3}(6 n)=\sum_{j=0}^{4} \sum_{\left(k_{1}, k_{2}\right) \in K_{j}^{3, n}} \overline{\theta_{n_{2}, n_{1}}^{\rho}} A_{j}\left(k_{1}, k_{2}\right) x^{k_{2} / 3} x^{\left(k_{1}+2 k_{2}\right) / 2},
\end{aligned}
$$


and

$$
G_{4}(6 n)=\sum_{j=0}^{4} \sum_{\left(k_{1}, k_{2}\right) \in K_{j}^{4, n}} \overline{\theta_{n_{2}, n_{1}}^{\rho}} g\left(n_{2}, p\right) A_{j}\left(k_{1}, k_{2}\right) x^{k_{2} / 3} x^{\left(k_{1}+2 k_{2}\right) / 2} .
$$

To expand $G_{i}(6(n+2))$, first an understanding of $K_{j}^{i, n+2}$ is needed. If $\left(k_{1}, k_{2}\right) \in$ $K_{j}^{i, n}$, then $\left(k_{1}, k_{2}+3\right) \in K_{j}^{i, n+2}$. For $i=3$ and $i=4$, this completely describes $K_{j}^{i, n+2}$, while the only elements of $K_{j}^{1, n+2}$ and $K_{j}^{2, n+2}$ that are not of this form are

$$
(3(n-j), 3) \in K_{j}^{1, n+2}
$$

and

$$
(3(n-1-j)+1,4) \in K_{j}^{2, n+2} .
$$

Now, using this description of $K_{j}^{i, n+2}$ and (5.13), write

$$
\begin{aligned}
G_{1}(6(n+2)) & =\sum_{j=0}^{4} \sum_{\left(k_{1}, k_{2}\right) \in K_{j}^{1, n}} \overline{\theta_{n_{2}, n_{1}}^{\rho}} A_{j}\left(k_{1}, k_{2}+3\right) x^{4} x^{\left(k_{1}+k_{2}\right) / 3} x^{\left(k_{1}+2 k_{2}\right) / 2} \\
& +\sum_{j=0}^{4} \overline{\theta_{n_{2}, n_{1}}^{\rho}} A_{j}(3(n-j), 3) x^{n-j} x^{3(n+2-j) / 2} .
\end{aligned}
$$

$$
\begin{aligned}
& G_{2}(6(n+2))= \sum_{j=0}^{4} \sum_{\left(k_{1}, k_{2}\right) \in K_{j}^{2, n}} \overline{\theta_{n_{2}, n_{1}}^{\rho}} g\left(n_{2}, p\right) A_{j}\left(k_{1}, k_{2}+3\right) \\
& \times x^{4} x^{\left(k_{1}+k_{2}-2\right) / 3} x^{\left(k_{1}+2 k_{2}\right) / 2} \\
&+\sum_{j=0}^{4} \overline{\theta_{n_{2}, n_{1}}^{\rho}} A_{j}(3(n-j-1)+1,4) x^{n-j} x^{3(n-j+2) / 2} .
\end{aligned}
$$

$$
G_{3}(6(n+2))=\sum_{j=0}^{4} \sum_{\left(k_{1}, k_{2}\right) \in K_{j}^{3, n}} \overline{\theta_{n_{2}, n_{1}}^{\rho}} A_{j}\left(k_{1}, k_{2}+3\right) x^{4} x^{k_{2} / 3} x^{\left(k_{1}+2 k_{2}\right) / 2} .
$$

$$
G_{4}(6(n+2))=\sum_{j=0}^{4} \sum_{\left(k_{1}, k_{2}\right) \in K_{j}^{4, n}} \overline{\theta_{n_{2}, n_{1}}^{\rho}} g\left(n_{2}, p\right) A_{j}\left(k_{1}, k_{2}+3\right) x^{4} x^{k_{2} / 3} x^{\left(k_{1}+2 k_{2}\right) / 2} .
$$

Now observe that for $k_{1} \geq 5$ and $k_{2} \geq 3$, Proposition 4.1 implies all the Gauss sums in (4.4) and (4.5) vanish. So for $n \geq 6$ it follows from the action of the Hecke operators that the coefficient of $a^{\rho}\left(n_{1} p^{k_{1}}, n_{2} p^{k_{2}}\right)$ in $A_{j}\left(k_{1}, k_{2}\right)$ is identical to the coefficient of $a^{\rho}\left(n_{1} p^{k_{1}}, n_{2} p^{k_{2}+3}\right)$ in $A_{j}\left(k_{1}, k_{2}+3\right)$. Therefore the proof of the claim is complete in the cases $i=3$ and $i=4$. To finish in the cases of $i=1$ and 
$i=2$ it remains to show that the second sums in both (5.18) and (5.19) vanish, or equivalently that

$$
\sum_{j=0}^{4} A_{j}(3(n-j), 3) x^{5(n-j) / 2}=0
$$

and

$$
\sum_{j=0}^{4} A_{j}(3(n-j-1)+1,4) x^{5(n-j) / 2}=0 .
$$

Explicitly,

$$
A_{0}(3 n, 3) x^{5 n / 2}=x^{5 n / 2} a^{\rho}\left(n_{1} p^{3 n}, n_{2} p^{3}\right),
$$

$$
\begin{aligned}
A_{1}(3 n-3,3) x^{5(n-1) / 2} & =-x^{5(n-1) / 2} x^{-2} \mu_{2} a^{\rho}\left(n_{1} p^{3 n-3}, n_{2} p^{3}\right) \\
& =-x^{5 n / 2}\left[a^{\rho}\left(n_{1} p^{3 n-6}, n_{2} p^{3}\right)+a^{\rho}\left(n_{1} p^{3 n}, n_{2}\right)\right. \\
& \left.+a^{\rho}\left(n_{1} p^{3 n-6}, n_{2} p^{6}\right)+a^{\rho}\left(n_{1} p^{3 n}, n_{1} p^{3}\right)\right],
\end{aligned}
$$

$$
\begin{aligned}
A_{2}(3 n-6,3) x^{5(n-2) / 2} & =x^{5(n-2) / 2}\left[x^{-1} \mu_{1}+x^{3}+x^{5}\right] a^{\rho}\left(n_{1} p^{3 n-6}, n_{2} p^{3}\right) \\
= & x^{5 n / 2}\left[a^{\rho}\left(n_{1} p^{3 n}, n_{2}\right)+a^{\rho}\left(n_{1} p^{3 n-6}, n_{2}\right)\right. \\
& +2 a^{\rho}\left(n_{1} p^{3 n-6}, n_{2} p^{3}\right)+a^{\rho}\left(n_{1} p^{3 n-6}, n_{2} p^{6}\right) \\
& \left.+a^{\rho}\left(n_{1} p^{3 n-12}, n_{2} p^{6}\right)\right],
\end{aligned}
$$

$$
\begin{aligned}
A_{3}(3 n-9,3) x^{5(n-3) / 2} & =-x^{5(n-3) / 2} x^{2} \mu_{2} a^{\rho}\left(n_{1} p^{3 n-9}, n_{2} p^{3}\right) \\
& =-x^{5 n / 2}\left[a^{\rho}\left(n_{1} p^{3 n-12}, n_{2} p^{3}\right)+a^{\rho}\left(n_{1} p^{3 n-6}, n_{2}\right)\right. \\
& \left.+a^{\rho}\left(n_{1} p^{3 n-12}, n_{2} p^{6}\right)+a^{\rho}\left(n_{1} p^{3 n-6}, n_{2} p^{3}\right)\right],
\end{aligned}
$$

and

$$
\begin{aligned}
A_{1}(3(n-2)+1,4) x^{5(n-1) / 2} & =-\mu_{2} x^{-2} x^{5(n-1) / 2} a^{\rho}\left(n_{1} p^{3 n-5}, n_{2} p^{4}\right) \\
& =-x^{5 n / 2}\left[a^{\rho}\left(n_{1} p^{3 n-8}, n_{2} p^{4}\right)+a^{\rho}\left(n_{1} p^{3 n-8}, n_{2} p^{7}\right)\right. \\
& \left.+a^{\rho}\left(n_{1} p^{3 n-2}, n_{2} p\right)+a^{\rho}\left(n_{1} p^{3 n-2}, n_{2} p^{4}\right)\right],
\end{aligned}
$$

$$
\begin{aligned}
A_{2}(3(n-3)+1,4) x^{5(n-2) / 2} & =\left[\mu_{1} x^{-1}+x^{3}+x^{5}\right] a^{\rho}\left(n_{1} p^{3 n-8}, n_{2} p^{4}\right) x^{5(n-2) / 2} \\
& =x^{5 n / 2}\left[a^{\rho}\left(n_{1} p^{3 n-14}, n_{2} p^{7}\right)+a^{\rho}\left(n_{1} p^{3 n-8}, n_{2} p\right)\right. \\
& +2 a^{\rho}\left(n_{1} p^{3 n-8}, n_{2} p^{4}\right)+a^{\rho}\left(n_{1} p^{3 n-8}, n_{2} p^{7}\right) \\
& \left.+a^{\rho}\left(n_{1} p^{3 n-2}, n_{2} p\right)\right]
\end{aligned}
$$


(5.32)

$$
\begin{aligned}
& A_{3}(3(n-4)+1,4) x^{5(n-3) / 2}=-\mu_{2} x^{3} a^{\rho}\left(n_{1} p^{3 n-11}, n_{2} p^{4}\right) x^{5(n-3) / 2} \\
& -x^{5 n / 2}\left[a^{\rho}\left(n_{1} p^{3 n-14}, n_{2} p^{4}\right)+a^{\rho}\left(n_{1} p^{3 n-14}, n_{2} p^{7}\right)\right. \\
& \left.+a^{\rho}\left(n_{1} p^{3 n-8}, n_{2} p\right)+a^{\rho}\left(n_{1} p^{3 n-8}, n_{2} p^{4}\right)\right] \text {, } \\
& A_{4}(3(n-5)+1,4) x^{5(n-4) / 2}=x^{5 n / 2} a^{\rho}\left(n_{1} p^{3 n-14}, n_{2} p^{4}\right) .
\end{aligned}
$$

Now summing (5.24)-(5.28) yields (5.22) and summing (5.29)-(5.33) yields (5.23). Now to finish the proof of Lemma 5.2, it remains to show (5.5) in the cases $n=6$ and $n=7$. But due to (5.6) this is equivalent to showing $G(36)=0$ and $G(42)=0$. These two facts can be verified by using (5.4), as was done with $G(0), G(6), G(12), G(18), G(24)$, and $G(30)$. The additional information needed from Theorem 4.2 is:

$$
\begin{aligned}
& \mu_{1} G^{\prime}(30) \\
& =\overline{\theta_{n_{2}, n_{1}}^{\rho}}\left[x^{33 / 2} a_{n_{1} p^{3}, n_{2} p^{3}}^{\rho}+\left(x^{3}+x^{2}+x-1\right) x^{29 / 2} a_{n_{1} p^{3}, n_{2} p^{6}}^{\rho}\right. \\
& +x^{33 / 2} a_{n_{1} p^{3}, n_{2} p^{9}}^{\rho}+x^{35 / 2} a_{n_{1} p^{9}, n_{2}}^{\rho}+\left(x^{3}+x^{2}+x-1\right) x^{31 / 2} a_{n_{1} p^{9}, n_{2} p^{3}}^{\rho} \\
& +x^{35 / 2} a_{n_{1} p^{9}, n_{2} p^{6}}^{\rho}+\left(x^{2}+x-1\right) x^{33 / 2} a_{n_{1} p^{15}, n_{2}}^{\rho}+x^{37 / 2} a_{n_{1} p^{15}, n_{2} p^{3}}^{\rho} \\
& +x^{35 / 2} a_{n_{1} p^{11}, n_{2} p}^{\rho} \overline{g\left(n_{1}, p\right)}+x^{35 / 2} a_{n_{1} p^{17}, n_{2} p}^{\rho} \overline{g\left(n_{1}, p\right)} \\
& +x^{31 / 2} a_{n_{1} p, n_{2} p^{4}}^{\rho} g\left(n_{1}, p\right)+\left(x^{3}+x^{2}+x-1\right) x^{27 / 2} a_{n_{1} p, n_{2} p^{7}}^{\rho} g\left(n_{1}, p\right) \\
& +x^{31 / 2} a_{n_{1} p, n_{2} p^{10}}^{\rho} g\left(n_{1}, p\right)+x^{33 / 2} a_{n_{1} p^{7}, n_{2} p}^{\rho} g\left(n_{1}, p\right) \\
& +\left(x^{3}+x^{2}+x-1\right) x^{29 / 2} a_{n_{1} p^{7}, n_{2} p^{4}}^{\rho} g\left(n_{1}, p\right)+x^{33 / 2} a_{n_{1} p^{7}, n_{2} p^{7}}^{\rho} g\left(n_{1}, p\right) \\
& +\left(x^{2}+x-1\right) x^{31 / 2} a_{n_{1} p^{13}, n_{2} p}^{\rho} g\left(n_{1}, p\right)+x^{35 / 2} a_{n_{1} p^{13}, n_{2} p^{4}}^{\rho} g\left(n_{1}, p\right) \\
& \left.+x^{33 / 2} a_{n_{1} p^{11}, n_{2}}^{\rho} g\left(n_{1}, p\right)^{2}+x^{33 / 2} a_{n_{1} p^{17}, n_{2}}^{\rho} g\left(n_{1}, p\right)^{2}\right] \text {, }
\end{aligned}
$$

$\mu_{2} G^{\prime}(30)$

$$
\begin{aligned}
= & \overline{\theta_{n_{2}, n_{1}}^{\rho}}\left[(x+1) x^{14} a_{n_{1}, n_{2} p^{6}}^{\rho}+(x+1) x^{14} a_{n_{1}, n_{2} p^{9}}^{\rho}+(x+1) x^{15} a_{n_{1} p^{6}, n_{2} p^{3}}^{\rho}\right. \\
& +(x+1) x^{15} a_{n_{1} p^{6}, n_{2} p^{6}}^{\rho}+(x+1) x^{16} a_{n_{1} p^{12}, n_{2}}^{\rho}+(x+1) x^{16} a_{n_{1} p^{12}, n_{2} p^{3}}^{\rho} \\
& +x^{17} a_{n_{1} p^{18}, n_{2}}^{\rho}+x^{16} a_{n_{1} p^{14}, n_{2} p}^{\rho} \overline{g\left(n_{1}, p\right)}+(x+1) x^{14} a_{n_{1} p^{4}, n_{2} p^{4}}^{\rho} g\left(n_{1}, p\right) \\
& +(x+1) x^{14} a_{n_{1} p^{4}, n_{2} p^{7}}^{\rho} g\left(n_{1}, p\right)+(x+1) x^{15} a_{n_{1} p^{10}, n_{2} p}^{\rho} g\left(n_{1}, p\right) \\
& +(x+1) x^{15} a_{n_{1} p^{10}, n_{2} p^{4}}^{\rho} g\left(n_{1}, p\right)+x^{16} a_{n_{1} p^{16}, n_{2} p}^{\rho} g\left(n_{1}, p\right) \\
& \left.+x^{15} a_{n_{1} p^{14}, n_{2}}^{\rho} g\left(n_{1}, p\right)^{2}\right],
\end{aligned}
$$

$G^{\prime}(36)$

$$
\begin{aligned}
= & \overline{\theta_{n_{2}, n_{1}}^{\rho}}\left[x^{12} a_{n_{1}, n_{2} p^{9}}^{\rho}+x^{13} a_{n_{1} p^{6}, n_{2} p^{6}}^{\rho}+x^{14} a_{n_{1} p^{12}, n_{2} p^{3}}^{\rho}+x^{15} a_{n_{1} p^{18}, n_{2}}^{\rho}\right] \\
& \left.+x^{12} a_{n_{1} p^{4}, n_{2} p^{7}}^{\rho} g\left(n_{1}, p\right)+x^{13} a_{n_{1} p^{10}, n_{2} p^{4}}^{\rho} g\left(n_{1}, p\right)+x^{14} a_{n_{1} p^{16}, n_{2} p}^{\rho} g\left(n_{1}, p\right)\right],
\end{aligned}
$$


$\mu_{2} G^{\prime}(36)$

$$
\begin{aligned}
= & \overline{\theta_{n_{2}, n_{1}}^{\rho}}\left[(x+1) x^{33 / 2} a_{n_{1} p^{3}, n_{2} p^{6}}^{\rho}+(x+1) x^{33 / 2} a_{n_{1} p^{3}, n_{2} p^{9}}^{\rho}\right. \\
& +(x+1) x^{35 / 2} a_{n_{1} p^{9}, n_{2} p^{3}}^{\rho}+(x+1) x^{35 / 2} a_{n_{1} p^{9}, n_{2} p^{6}}^{\rho}+(x+1) x^{37 / 2} a_{n_{1} p^{15}, n_{2}}^{\rho} \\
& +(x+1) x^{37 / 2} a_{n_{1} p^{15}, n_{2} p^{3}}^{\rho}+x^{39 / 2} a_{n_{1} p^{21}, n_{2}}^{\rho}+x^{37 / 2} a_{n_{1} p^{17}, n_{2} p}^{\rho} \overline{g\left(n_{1}, p\right)} \\
& +(x+1) x^{31 / 2} a_{n_{1} p, n_{2} p^{7}}^{\rho} g\left(n_{1}, p\right)+(x+1) x^{31 / 2} a_{n_{1} p, n_{2} p^{10}}^{\rho} g\left(n_{1}, p\right) \\
& +(x+1) x^{33 / 2} a_{n_{1} p^{7}, n_{2} p^{4}}^{\rho} g\left(n_{1}, p\right)+(x+1) x^{33 / 2} a_{n_{1} p^{7}, n_{2} p^{7}}^{\rho} g\left(n_{1}, p\right) \\
& +(x+1) x^{35 / 2} a_{n_{1} p^{13}, n_{2} p}^{\rho} g\left(n_{1}, p\right)+(x+1) x^{35 / 2} a_{n_{1} p^{13}, n_{2} p^{4}}^{\rho} g\left(n_{1}, p\right) \\
& \left.+x^{37 / 2} a_{n_{1} p^{19}, n_{2} p}^{\rho} g\left(n_{1}, p\right)+x^{35 / 2} a_{n_{1} p^{17}, n_{2}}^{\rho} g\left(n_{1}, p\right)^{2}\right],
\end{aligned}
$$

$G^{\prime}(42)$

$$
\begin{aligned}
= & \overline{\theta_{n_{2}, n_{1}}^{\rho}}\left[x^{29 / 2} a_{n_{1} p^{3}, n_{2} p^{9}}^{\rho}+x^{31 / 2} a_{n_{1} p^{9}, n_{2} p^{6}}^{\rho}+x^{33 / 2} a_{n_{1} p^{15}, n_{2} p^{3}}^{\rho}\right. \\
& +x^{35 / 2} a_{n_{1} p^{21}, n_{2}}^{\rho}+x^{27 / 2} a_{n_{1} p, n_{2} p^{10}}^{\rho} g\left(n_{1}, p\right)+x^{29 / 2} a_{n_{1} p^{7}, n_{2} p^{7}}^{\rho} g\left(n_{1}, p\right) \\
& \left.+x^{31 / 2} a_{n_{1} p^{13}, n_{2} p^{4}}^{\rho} g\left(n_{1}, p\right)+x^{33 / 2} a_{n_{1} p^{19}, n_{2} p}^{\rho} g\left(n_{1}, p\right)\right] .
\end{aligned}
$$

As an immediate consequence of Lemma 5.2, Theorem 5.1 follows by pulling out the Euler factors in $R(s)$. The fact that the Euler product has a meromorphic continuation to the whole complex plane follows from the meromorphic continuation of the Eisenstein series $E(\hat{f}, \tau, \bar{s})$.

In order to prove a functional equation for the Euler product, Theorem 3.1 and Theorem 5.1 need to be extended to the situation where $\phi$ and $\Theta$ are replaced with non-metaplectic minimal parabolic Eisenstein series on GSp(4) and GL(3) respectively. As this has not yet been carried out, the rest of the section serves only as a suggestion of what the author believes the functional equation will be.

Suppose $\mu_{1}, \mu_{2}, \nu_{1}, \nu_{2}$ are complex numbers and define

$$
\alpha_{1}=2 \mu_{1}+\mu_{2}-\frac{3}{2}, \quad \alpha_{2}=\mu_{2}-\frac{1}{2}
$$

and

$$
\beta_{1}=1-\frac{\nu_{1}}{2}-\nu_{2}, \quad \beta_{2}=\frac{-\nu_{1}}{2}+\frac{\nu_{2}}{2}, \quad \beta_{3}=\nu_{1}+\frac{\nu_{2}}{2}-1 .
$$

Also define

$$
L(s, \phi)=3^{-2 s / 3}(2 \pi)^{-4 s} \Gamma\left(s-3 \alpha_{1}\right) \Gamma\left(s-3 \alpha_{2}\right) \Gamma\left(s+3 \alpha_{1}\right) \Gamma\left(s+3 \alpha_{2}\right) R(s) R_{\lambda}(s),
$$

where $R(s), R_{\lambda}(s)$ are defined as in Theorem 5.1. Then, assuming that the RankinSelberg integral in Theorem 3.1 can be extended to non-cusp forms, arguments similar to those in Chapter 10 of [B1] may be used to deduce a local functional equation. By use of the triplication formula for the $\Gamma$-function (compare the use of the duplication formula in [FrW]) it should be possible to show that $L(s, \phi)$ possesses a functional equation as $s \rightarrow 1-s$.

\section{REFERENCES}

[BMS] H. Bass, J. Milnor, J.P. Serre, Solution of the congruence subgroup problem for $\mathrm{SL}_{n}(n \geq$ 3) and $\mathrm{Sp}_{2 n}(n \geq 2)$, Publ. Math., IHES 33, 59-137 (1967). MR 39:5574 
[B1] D. Bump, Automorphic Forms on $\mathrm{GL}(3, \mathbb{R})$, Springer Lecture Notes in Mathematics, 1083 (1984). MR 86g:11028

[B2] D. Bump, The Rankin-Selberg Method: A Survey. In: Number theory, trace formulas and discrete groups: a symposium in honor of Atle Selberg, Academic Press, (1989), pp. 49-109. MR 90m:11070

[BFrH1] D. Bump, S. Friedberg, J. Hoffstein, Eisenstein series on the metaplectic group and nonvanishing theorems for automorphic L-functions and their derivatives, Annals of Math. 131, 53-127 (1990). MR 92e: 11053

[BFrH2] D. Bump, S. Friedberg, J. Hoffstein, The Kubota symbol for $S p(4, \mathbb{Q}[i])$, Nagoya Math. J. 119, 173-188 (1990). MR 91m:11036

[BH1] D. Bump, J. Hoffstein, Cubic metaplectic forms on GL(3), Inventiones Math. 84, 481-505 (1986). MR 87i:11059

[BH2] D. Bump, J. Hoffstein, Some Euler products associated with cubic metaplectic forms on GL(3), Duke Math. J. 53, 1047-1072 (1986). MR 88d:11044

[BH3] D. Bump, J. Hoffstein, On Shimura's correspondence, Duke Math. J. 55, 661-691 (1987). MR 89c: 11072

[CF] J. Cassels, A. Fröhlich, Algebraic Number Theory, Academic Press, 1967. MR 35:6500

[Fli] Y. Flicker, Automorphic forms on covering groups of $G L(2)$, Invent. Math. 57 no. 2, 119-182 (1980). MR 81m:10057

[FliKa] Y. Flicker, D. Kazhdan Metaplectic Correspondence, Publ. Math., IHES 64, 53-110 (1986). MR 88d:11049

[Fr] S. Friedberg, (1,2,1) Coset Representatives on $S p(4)$, Handwritten notes (1988).

[FrW] S. Friedberg, S. Wong, On the Shimura correspondence for GSp(4), Math. Ann. 290, 183-207 (1991). MR 92f:11063

[GeP-S] S. Gelbart, I. Piatetski-Shapiro, L-functions for $G \times G L(n)$, Part B in Springer Lecture Notes in Mathematics, 1254 (1985). MR 89k:11038

[Gi] D. Ginzburg, L-functions for $S O_{n} \times G L_{k}$, J. Reine Angew. Math. 405 (1990), 156-180. MR 91g:11048

[KaP1] D. Kazhdan, S. Patterson, Metaplectic Forms, Publ. Math., IHES 59, 35-142 (1984). MR 85g:22033; MR 87h:22024

[KaP2] D. Kazhdan, S. Patterson, Towards a generalized Shimura correspondence, Advances in Math. 60, 161-234 (1986). MR 87 m:22050

[Ku] T. Kubota, Automorphic Forms and Reciprocity in a Number Field, Kiyokuniya Book Store, 1969.

[La] R. Langlands, Euler Products, Yale Mathematical Monographs 1 (1971). MR 54:7387

$[\mathrm{Pa}] \quad$ S. Patterson, A cubic analogue of the theta series, J. Reine und Angew. Math. 296 125-161 (1977). MR 58:27795a

[P-S] I. Piatetski-Shapiro, Euler Subgroups. In: Lie Groups and their Representations, John Wiley and Sons (1975), pp. 597-620. MR 53:10720

[Pr] N. Proskurin, Automorphic functions and the Bass-Milnor-Serre homomorphism, I, II, Zap. Nauch. Sem. LOMI, 129, 85-126, 127-163 (1983) (translation from Russian to English: J. of Sov. Math. 29 1160-1191, 1192-1218 (1985)). MR 85e:11035

[Sa] G. Savin, Local Shimura correspondence, Math. Ann. 280, 185-190 (1988). MR 89h:22018

[Shal] J. Shalika, The multiplicity one theorem for GL(n), Annals of Math 100 (1974), 171-193. MR 50:545

[Shi] G. Shimura, On modular forms of half-integral weight, Ann. Math. 97, 440-481 (1973). MR 48:10989

[So] D. Soudry, Rankin-Selberg Convolutions for $S O_{2 l+1} \times G L_{n}$ : Local Theory, Memoirs of the A.M.S. \#500 (1993). MR 94b:11043

IBM, 4000 Executive Parkway Suite 300, San Ramon, California 94583

Current address: Pacific Bell, 2600 Camino Ramon 15200W, San Ramon, California 94583

E-mail address: tegoetz@srv.pacbell.com 